Article

\title{
Effects of Bogies on the Wake Flow of a High-Speed Train
}

\author{
Wen Liu ${ }^{1,2}$, Dilong Guo ${ }^{1,2, *}$, Zijian Zhang ${ }^{1,2}$, Dawei Chen ${ }^{3}$ and Guowei Yang ${ }^{1,2}$ \\ 1 School of Engineering Science, University of Chinese Academy of Sciences, Beijing 100049, China; \\ liuwen2@imech.ac.cn (W.L.); zhangzijian@imech.ac.cn (Z.Z.); gwyang@imech.ac.cn (G.Y.) \\ 2 Institute of Mechanics, Chinese Academy of Sciences, Beijing 100190, China \\ 3 CRRC Qingdao Sifang Co., Ltd., Qingdao 266111, China; chendawei@cqsf.com \\ * Correspondence: Jack9517@126.com; Tel.: +86-10-8254-3815
}

Received: 30 December 2018; Accepted: 13 February 2019; Published: 21 February 2019

check for updates

\begin{abstract}
The wake region of high-speed trains is an area of complex turbulent flow characterized by the periodic generation and shedding of vortices, which causes discomfort to passengers and affects the stability and safety of the train. In this study, the unsteady characteristics of the wake flows of three 1:1 scale China Railway High-Speed 380A (CRH380A) high-speed train models with different degrees of simplification were numerically investigated using the improved delayed detached eddy simulation (IDDES) method. Analyses of the aerodynamic forces, train-induced slipstream, and turbulent kinetic energy (TKE) were conducted to determine the effects of the bogies on the wake flow of the high-speed train. It was found that the existence of bogies on the bottom of the train, especially the last bogie, not only enhanced the wake flow but also introduced large perturbances into the wake flow. Moreover, the generation and evolution of the vortices in the wake flows were determined by analyzing the instantaneous flow fields and coherent flow structures that were obtained by the dynamic mode decomposition (DMD) method. The results showed that a pair of large, counter-rotating streamwise vortices in the real model of the high-speed train was generated by the cowcatcher and their intensity was significantly enhanced by perturbances that were introduced by the bogies on the bottom of the train.
\end{abstract}

Keywords: high-speed train; unsteady wake; bogie; DMD; vortex dynamics

\section{Introduction}

The wake region of high-speed trains is an area of very complex turbulent flow. Vortices of different strengths are rapidly generated and shed, especially in the near wake area, which has adverse effects on high-speed trains: not only does it increase the pressure drag, but also seriously affects the lift and side forces of the trailing car. This problem causes discomfort to the passengers and affects the stability and safety of the train [1-7]. Therefore, the optimization of the wake flow is an important aspect of high-speed train research. It is imperative to understand the detailed mechanism of the generation of trailing vortices as a result of the complex geometry of high-speed trains.

Many studies have been conducted on the wake flow of high-speed trains. Baker et al. [1] conducted experiments on a 1/25-scale moving model rig to analyze the wakes and slipstreams of high-speed trains and to provide an understanding of the motions of the wake-vortex structures. Bell et al. [5-7] used Cobra probes, surface pressure measurements, and particle image velocimetry (PIV) flow visualization technique in wind tunnel tests to determine the topology structure of wake vortices of an ICE3 high-speed train; oscillations in the spanwise and vertical directions were obtained. Weise et al. [8] used oil paint and smoke visualization techniques in wind tunnel tests to analyze the near wake of high-speed trains; it was found that the type of flow structure, i.e., a separation 
bubble or vortex shedding, depended on the geometry of the trailing car. Yao et al. [9] compared the unsteady Reynolds average Navier-Stokes (URANS) and detached eddy simulation (DES) methods in wake-flow simulations of a high-speed train with different running speeds. The results of these studies indicated that the wake of a modern high-speed train is highly complex, three-dimensional, and consists of shear layers, separation and recirculation regions, and shedding vortices. The wake is always characterized by a pair of large counter-rotating streamwise vortices; the vortices move towards the ground and meanwhile separate and subsequently break up as a result of their mutual inductions and the interactions with the ground. The oscillations of the shedding vortices in both the spanwise and vertical directions of an ICE2 high-speed train were identified in URANS simulations by Schulte-Werning et al. [10]; it was found that the oscillations coincided with the periodic oscillations of the side and lift forces. Baker et al. [2,3] conducted full-scale train tests of an ICE2 train and determined that the dimensionless frequency of vortex shedding in the wake was $\mathrm{St}=0.11$ (St is the Strouhal number). Numerical simulations using an improved delayed detached eddy simulation (IDDES) were conducted by Xia et al. [11] to investigate the effects of the ground configurations on the near wake; the frequency in the wake was $\mathrm{St}=0.118$. Pii et al. [12] identified vortex shedding at frequencies of $\mathrm{St}=0.15-0.18$; the vortices originated from the underbody due to the interaction with the bogies, as determined by the Lattice Boltzmann Method (LBM). The aforementioned results show that the near wake of high-speed trains is characterized by strong periodicity, resulting in periodic oscillations of the side and lift forces and the vortex shedding frequency is in the range of $\mathrm{St}=0.085-0.21$ and depends on the geometry of the trailing car. Moreover, it has been demonstrated via full-scale experiments [3,4], wind tunnel experiments [5] and numerical simulations [13,14] that a strong slipstream is always induced by the highly unsteady wake flow and may endanger passengers waiting at platforms and track-side workers.

Aside from various methods for identifying the wake vortex structures, such as the PIV method in wind tunnel tests, the hot-wire anemometer (HWA) and surface pressure measurements in real trains, and moving-model tests and the instantaneous flow field analyses in numerical simulations, some modern decomposition methods based on the instantaneous flow field data have also been applied to flow structure identifications recently. Muld et al. [15] used the proper orthogonal decomposition (POD) and dynamic mode decomposition (DMD) methods to extract the wake-flow structures of the aerodynamic train model (ATM) high-speed trains; the authors identified a pair of counter-rotating streamwise vortices as the dominant wake feature and these vortices were periodically generated and shed at a frequency of $\mathrm{St}=0.085$. Bell et al. [6] analyzed the surface pressure of the ICE3 trailing car with the POD method and found that the vortex shedding frequency was $\mathrm{St}=0.2$. The coherent correlation of several dominant modes and the corresponding power spectral density distributions were also investigated. Xia et al. [16] decomposed the PIV data of the CRH3 high-speed train using the POD method and analyzed the coherent structures. Their results clarified the physical meaning of each mode and the dynamic characteristics of the near wake.

It is well known that high-speed trains have a unique geometry with a large number of complex and sophisticated accessory parts, such as bogies, pantographs, inter-carriage gaps, air conditioning, and so on, making it impossible to fully replicate the flow fields. Therefore, high-speed trains are always simplified to certain degrees in model tests and numerical simulations. For example, Bell et al. [6,7] used a 1/10th-scale model of an ICE3, which had four sets of bogies but no pantograph, no inter-carriage gap, and no air conditioning in wind-tunnel tests; Muld et al. $[15,17]$ used a $1 / 50$ th-scale model of an ATM in their numerical simulations with simple bogies but no pantographs. However, several studies $[9,12,18]$ have demonstrated that the accessory parts, especially the bogies, not only have large impacts on the nearby flows, but also on the wake of the train. However, the unique effects of bogies on the generation and evolution of vortices in the wake flow of high-speed trains are not fully understood. A better understanding of these effects will help researchers appropriately simplify high-speed train models in experiments or numerical simulations to investigate the wake flow of high-speed trains accurately and effectively. 
In this paper, the unsteady characteristics of the wake flows of three 1:1 China Railway High-Speed 380A (CRH380A) models with different degrees of simplification are numerically studied using the IDDES method. First, the numerical details are introduced in Section 2, followed by the description of the DMD method in Section 3 and the validations and grid convergence tests in Section 4. Next, the results of the analysis of the aerodynamic forces, train-induced slipstream, and turbulent kinetic energy (TKE) are presented in Sections 5.1 and 5.2 and we discuss the effects of the bogies on the wake flow of the high-speed train. The instantaneous wake flow fields and their coherent flow structures obtained by the DMD method are presented in Sections 5.3 and 5.4, respectively. Finally, the detailed mechanism of the generation and evolution of trailing vortices in a real CRH380A high-speed train is explained in Section 5.5.

\section{Computational Details}

\subsection{Computational Models and Domains}

The high-speed train model is a 1:1 scale of the CRH380A and consists of one head car, one middle car, and one trailing car. The head car and trailing car are symmetrical and have a length of $26.5 \mathrm{~m}$. The model dimensions are $78 \mathrm{~m} \times 3.5 \mathrm{~m} \times 3.36 \mathrm{~m}$ in length, height, and width, as shown in Figure 1 . In this study, three models with different degrees of simplification are used. Model 1, denoted as the real model, has a complex geometry of the bogies and inter-carriage gaps (Figure 1). Notably, the pantographs and air conditioning are ignored in this study because it was shown that they had a relatively small impact on the wake [9]. Model 2, denoted as the simplified model has a simplified geometry with no bogies and a floor, as shown in Figure 2. Model 3, denoted as the semi-simplified model, is based on model 2 and has a bogie near the nose of the trailing car, as shown in Figure 3. As known, different flow fields would be surely obtained with different train models, but their computational costs do not differ much. It should be emphasized here that the purpose of the three train models with different degrees of simplification of the bogies is not to discuss the computational costs of high-speed train simulations but to analyze the effects of the bogies on the generation and evolution of vortices in the wake flows of high-speed trains by comparing the three train models.

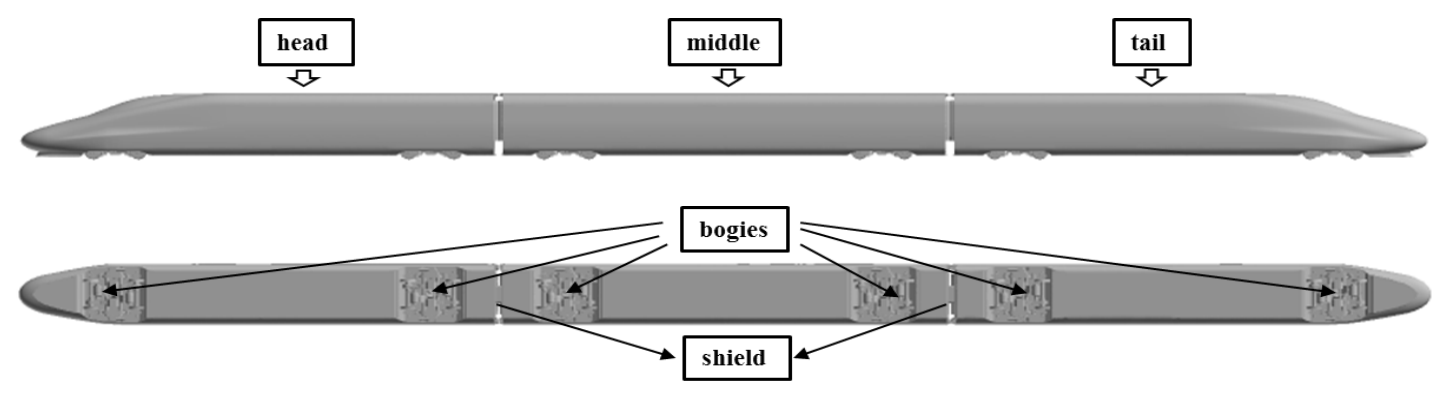

Figure 1. Real model.

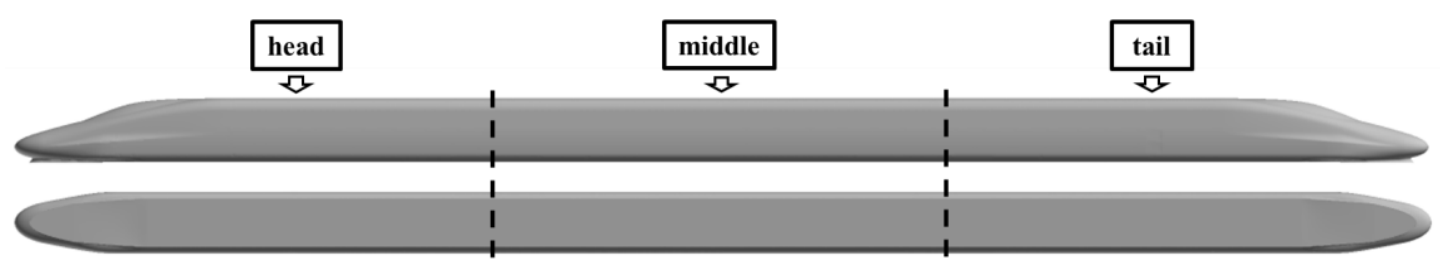

Figure 2. Simplified model. 


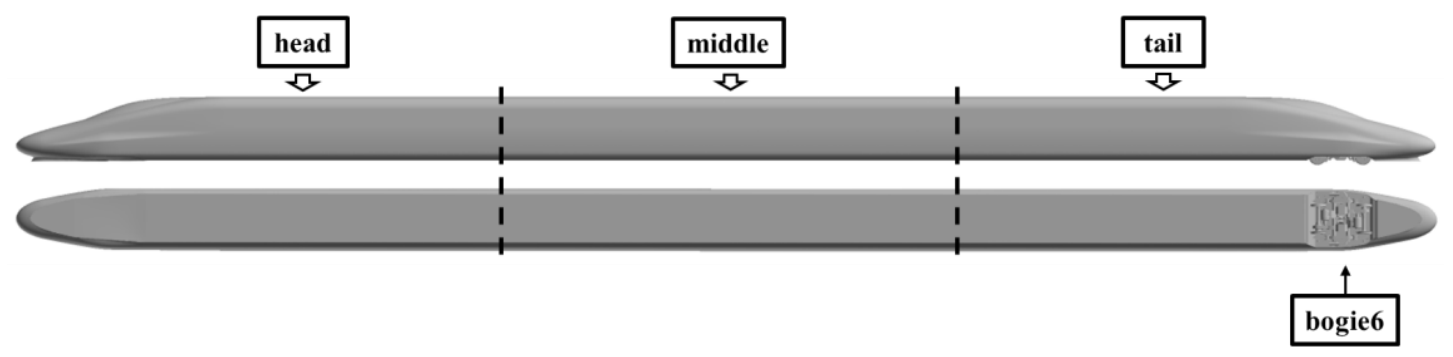

Figure 3. Semi-simplified model.

Let $\mathrm{H}$ denote the height of the train, $\mathrm{H}=3.5 \mathrm{~m}$. The computational domain extended $22.7 \mathrm{H}$ in front of the train and $46.4 \mathrm{H}$ behind the train. The top of the computational domain was at a distance of $12 \mathrm{H}$ from the bottom of the rail and the sides were at a distance of $8.57 \mathrm{H}$ from the center of the train, as shown in Figure 4. The same computational domain is used in the simulations of all three models.
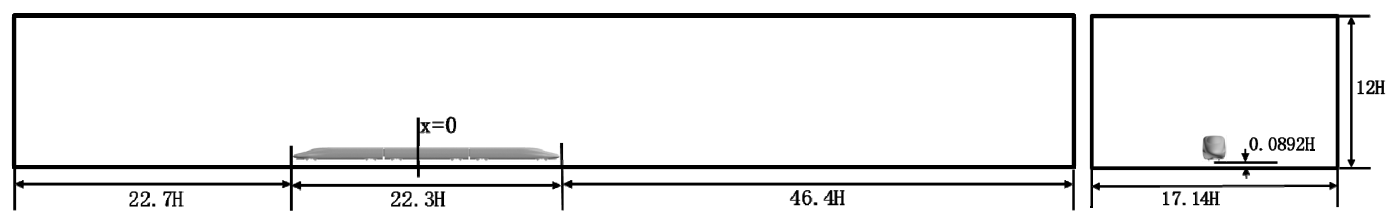

Figure 4. Computational domain.

We define the dead center of the train as $x=0, y=0$ and the bottom end of the wheels as $z=0$. The flow direction is defined as the positive direction of the $x$-axis, the vertical direction as the positive direction of the z-axis, and hence the positive direction of the $y$-axis can be determined by the right-hand rule. The coordinates of the front and back of the train are $(x, y, z)=(-39,0,1) \mathrm{m}$ and $(39,0$, 1) $m$, respectively.

A speed of $V=300 \mathrm{~km} / \mathrm{h}$ is set as the train speed for the simulations. The corresponding Mach number is 0.245 and hence the flow is in a compressible state. As a result, the inlet, outlet, and far-field of the computational domain are all set as non-reflective. A no-slip wall condition is used on the train surface to determine the frictional drag and pressure on the surface. The train is set as stationary and a moving wall boundary with the same speed as trains is used for the ground to simulate the ground effect caused by the relative motion between the train and the ground.

\subsection{Meshing Strategy}

Trimmed hexahedral grids are used in the outward zone of the train and prism layers are used near the wall area. To meet the usage requirement of the standard wall function (y+: 30-120), 10 prism layers are used on the wall surface and the height of the first layer is $2.29 \times 10^{-4} \mathrm{H}$. For a better connection with the hexahedral grids and to ensure good quality, the growth rate of the prism layer is set to 1.5 .

We use the real model (Figure 1) as an example of the grid layout; the grids on the cross-section of $y=0$ and on the surface of the trailing car are schematically shown in Figure 5. To accurately capture the details of the flow structures in the wake of the high-speed train, the grids are densified in the wake region and the maximum grid size in this zone is limited to $1.786 \times 10^{-2} \mathrm{H}$. In addition, the hexahedral grids are refined to create three zones around the train to ensure that the minimum grid size in the zones is limited to $3.571 \times 10^{-2} \mathrm{H}$. The grid topologies of the three models are kept as consistent as possible. As a result, the total grids contain about 54 million cells in model 1 and about 46 million cells in models 2 and 3. 

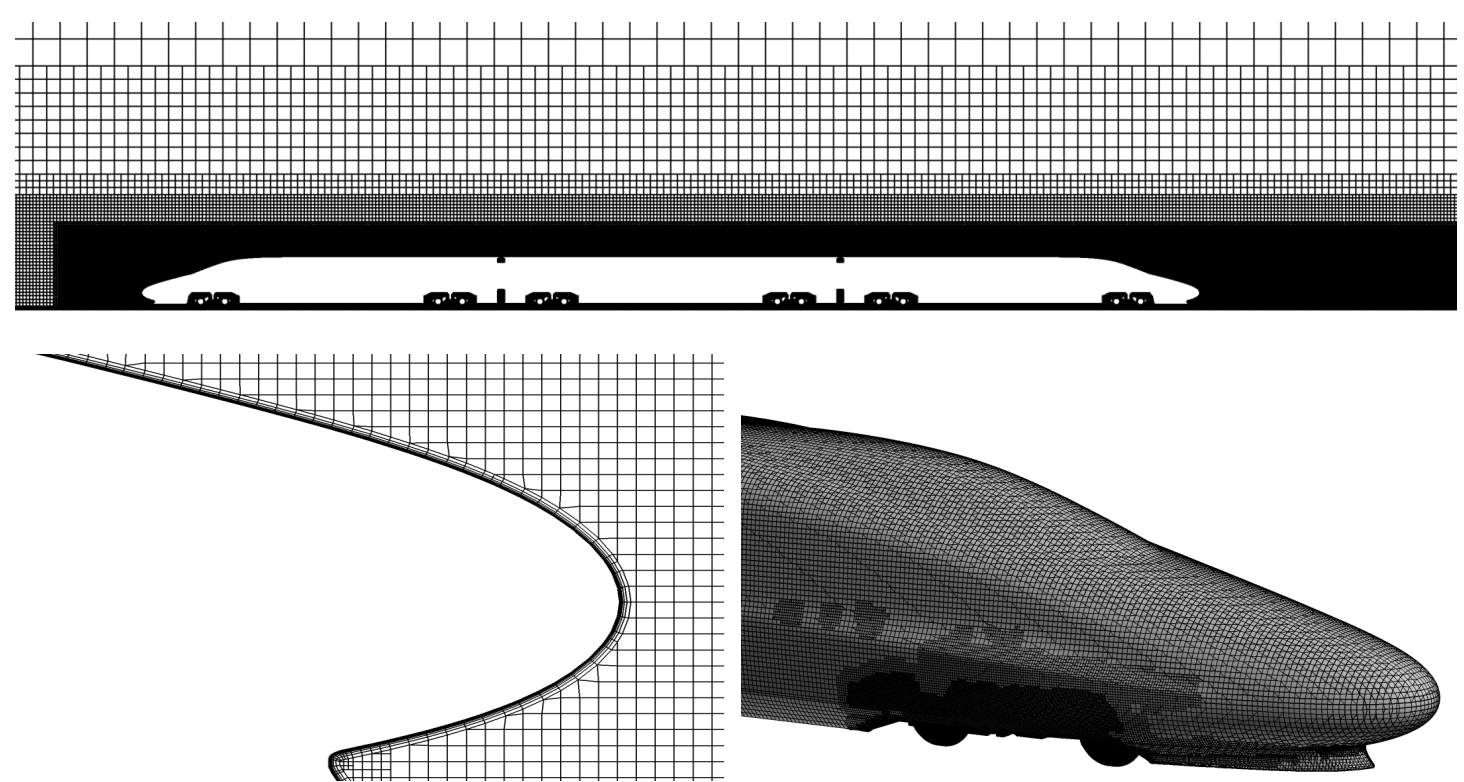

Figure 5. Grid layout on the cross-section of $y=0$ (upper) and on the surface of the trailing car (lower).

\subsection{Numerical Method}

Although numerical simulation methods in engineering applications are rather mature, difficulties still exist regarding the simulation of the real flow field of high-speed trains with complex geometry and large size. It is difficult to balance the computational cost and the accuracy of the results. The DES method is a hybrid method that combines the RANS method and large eddy simulation (LES) and has the advantages of being able to solve large separation problems with high Reynolds numbers. The DES method behaves as the RANS model close to the wall to simulate the small-scale pulsation with less calculation cost and as a LES model in regions away from the walls to simulate the motion of the large separation vortices with high accuracy. The IDDES method not only possesses the advantages of the DES but also prevents flow separations caused by modeling losses of turbulence stresses and grids. Therefore, the IDDES method is very suitable for the simulation of the complex wake of high-speed trains $[19,20]$.

The IDDES method based on the shear stress transport (SST) $k-\omega$ turbulence model is chosen to solve the compressible Navier-Stokes (N-S) equations in this study. The inviscid flux term is discretized using the Weiss-Smith preconditioned Roe's flux-difference splitting scheme and the viscous flux term is discretized using the second-order upwind scheme. Moreover, the transient term is discretized by using the Euler implicit two-order temporal scheme. A dual time-stepping technique that includes the use of inner iterations in pseudo-time is used to solve the unsteady flow equations; the physical time-step is set as $5 \times 10^{-4} \mathrm{~s}$ with 15 inner iterations to ensure that the residual value drops by at least one order of magnitude in each physical time step. All the computation results are obtained using STARCCM+. The criteria for determining that the flow around the train has been fully developed are that all residual errors cease to decrease in subsequent physical time steps and that the aerodynamic forces acting on the train exhibit stable fluctuations around the mean values.

\section{Dynamic Mode Decomposition}

Dynamic mode decomposition (DMD) is a data processing algorithm that extracts coherent structures with a single temporal frequency from a numerical or experimental data sequence. For the instantaneous flow field at time $i$, the samples are obtained at $M$ spatial locations and arranged in a certain order into a column vector $\psi_{i}$, which is called a snapshot of the flow field. We focus on the 
temporal evolution processes and assume that the data are equispaced in time and the time step is $\Delta t$. Next, we form two data matrices from $N+1$ snapshots:

$$
\left\{\begin{array}{c}
\boldsymbol{\Psi}_{0}=\left[\begin{array}{llll}
\boldsymbol{\psi}_{0} & \boldsymbol{\psi}_{1} & \cdots & \boldsymbol{\psi}_{N-1}
\end{array}\right] \\
\boldsymbol{\Psi}_{1}=\left[\begin{array}{llll}
\boldsymbol{\psi}_{1} & \boldsymbol{\psi}_{2} & \cdots & \boldsymbol{\psi}_{N}
\end{array}\right]
\end{array}\right.
$$

Assume that the snapshots are generated by a discrete-time linear time-invariant system:

$$
\psi_{t+1}=\mathbf{A} \psi_{t}, t=0,1 \cdots, N-1,
$$

resulting in:

$$
\mathbf{\Psi}_{1}=\left[\begin{array}{llll}
\psi_{1} & \psi_{2} & \cdots & \psi_{N}
\end{array}\right]=\left[\mathbf{A} \psi_{0} \mathbf{A} \psi_{1} \cdots \mathbf{A} \psi_{N-1}\right]=\mathbf{A} \Psi_{0} .
$$

In Equations (2) and (3), the characteristic information of the flow field is contained in matrix $\mathbf{A}$ and hence the evolution of the flow field can be obtained through an eigen decomposition of $\mathbf{A}$. However, in the flow field $M$, the number of sample points is always large so that $\mathbf{A} \in \mathbf{C}^{M \times M}$ becomes a high-dimensional matrix. The eigen decomposition of such a high-dimensional matrix is impossible to solve in practical applications. In DMD [21,22], the characteristic information is approximated by an alternative low-dimensional matrix $\mathbf{F}_{\mathrm{dmd}} \in \mathbf{C}^{N \times N}$. N, the number of snapshots, is always smaller than $M$ by 1-2 orders of magnitude, which results in lower calculation costs for the eigen decomposition.

Notably, all DMD calculations are performed in the complex field. First, $\Psi_{0}$ is decomposed using an economy-size singular value decomposition (SVD):

$$
\mathbf{\Psi}_{0}=\mathbf{U} \Sigma \mathbf{V}^{*}
$$

where * denotes the complex conjugate transpose of the matrix. Then, the optimal low-dimensional approximation matrix $\mathbf{F}_{\mathrm{dmd}}$ is determined by:

$$
\mathbf{F}_{\mathrm{dmd}}=\mathbf{U}^{*} \mathbf{\Psi}_{1} \mathbf{V} \Sigma^{-1}
$$

To calculate the DMD modes and corresponding eigenvalues of the flow field, the eigen decomposition of $\mathbf{F}_{\mathrm{dmd}}$ is required, i.e.:

$$
\mathbf{F}_{\mathrm{dmd}} \boldsymbol{y}_{k}=\mu_{k} \boldsymbol{y}_{k}, k=1,2, \cdots N .
$$

where $\boldsymbol{y}_{k}$ is the $k$ th eigenvector of $\mathbf{F}_{\mathrm{dmd}}$ and $\mu_{k}$ is the corresponding $k$ th eigenvalue. Thereafter, the DMD modes at $t=0$ can be obtained by:

$$
\boldsymbol{\varphi}_{k}^{(0)}=\mathbf{U} \boldsymbol{y}_{k} .
$$

In Equation (7), all modes are sorted in order of descending mode energy $\left\|\varphi_{k}^{(0)}\right\|$ as:

$$
\left\|\boldsymbol{\varphi}_{1}^{(0)}\right\|>\left\|\boldsymbol{\varphi}_{2}^{(0)}\right\|>\left\|\boldsymbol{\varphi}_{3}^{(0)}\right\|>\cdots>\left\|\boldsymbol{\varphi}_{N}^{(0)}\right\|,
$$

where $\left\|\boldsymbol{\varphi}_{k}^{(0)}\right\|$ can be evaluated by the following matrix norm [21,23],

$$
\left\|\boldsymbol{\varphi}_{k}^{(0)}\right\|=\frac{1}{\operatorname{norm}\left(\mathbf{V}^{*} \Sigma^{-1} \boldsymbol{y}_{k}\right)} .
$$


In Equation (6), the eigenvalue $\mu_{k}$, corresponding to the $k$ th DMD mode, represents the characteristic information in the unsteady flow field and the corresponding mode frequency $\omega_{k}$ and growth rate $g_{k}$ are:

$$
\left\{\begin{array}{l}
\omega_{k}=\operatorname{Im}\left[\frac{\ln \left(\mu_{k}\right)}{\Delta t}\right] . \\
g_{k}=\operatorname{Re}\left[\frac{\ln \left(\mu_{k}\right)}{\Delta t}\right]
\end{array} .\right.
$$

Thereafter, the $k$-order DMD mode at time $n$ can be calculated by:

$$
\boldsymbol{\varphi}_{k}^{(n)}=\mu_{k}^{n} \boldsymbol{\varphi}_{k}^{(0)} .
$$

The $k$-order reconstructed flow field at time $n$ can be calculated by:

$$
\boldsymbol{\Phi}_{k}^{(n)}=\sum_{i=0}^{k} \mu_{i}^{n} \boldsymbol{\varphi}_{i}^{(0)}
$$

\section{Method Validation}

\subsection{Grid Independence}

In this section, convergence tests are conducted to exclude the influence of the mesh resolution. For a comparison with the experimental data, model 1 is used as an example and three different mesh resolutions are investigated. All other computational details were explained in Section 2. The details of the three different meshes are shown in Table 1 . The simulation results of the aerodynamic characteristics using these meshes are compared with those obtained from a wind tunnel test. The test model used in the wind tunnel experiments is a 1/8th scaled model of the modified CRH380A train with a 3-car grouping, including the head car, middle car, and trailing car; the length of the model is $9.75 \mathrm{~m}$. The experiment was conducted at the China Aerodynamics Research and Development Center (CARDC) in Sichuan. The free stream velocity is $60 \mathrm{~m} / \mathrm{s}$. The experimental setups in the wind-tunnel

\begin{tabular}{|c|c|c|c|}
\hline & $\begin{array}{l}\text { First Thickness of Mesh } \\
\text { Close to Train Surface }\end{array}$ & $\begin{array}{c}\text { Refine Mesh Near } \\
\text { Accessory Parts (size) }\end{array}$ & $\begin{array}{l}\text { Total Number of } \\
\text { Mesh Cells }\end{array}$ \\
\hline FM & $1.14 \times 10^{-4} \mathrm{H}$ & Yes $\left(4.46 \times 10^{-3} \mathrm{H}\right)$ & 74 million \\
\hline MM & $2.29 \times 10^{-4} \mathrm{H}$ & Yes $\left(8.93 \times 10^{-3} \mathrm{H}\right)$ & 54 million \\
\hline $\mathrm{CM}$ & $4.57 \times 10^{-4} \mathrm{H}$ & No & 37 million \\
\hline
\end{tabular}
are shown in Figure 6.

Table 1. Details of the three meshes (FM: fine mesh, MM: middle mesh, CM: coarse mesh).

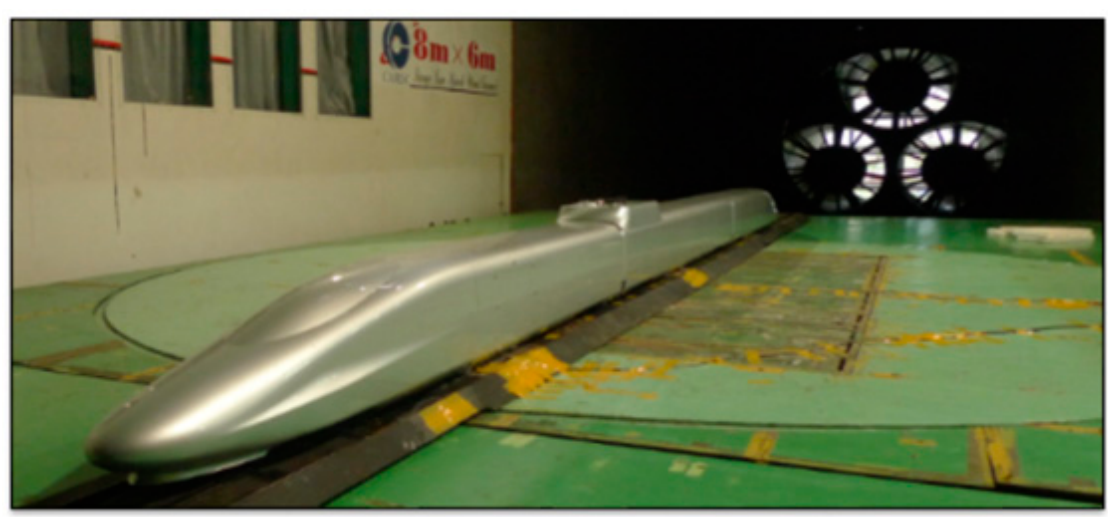

Figure 6. Wind tunnel test setup at CARDC, Sichuan. 
Aerodynamic coefficients are often used for a comparison of models with different scales. The drag coefficient $C_{d}$ is defined in Equation (13); the results are summarized in Table 2.

$$
C_{d}=\frac{F_{d}}{0.5 \rho V^{2} S}
$$

where $F_{d}$ is the drag force, $\rho$ is the air density, and $S$ is a reference area, in this case, the train's cross-sectional area (in this study, $S=11.123 \mathrm{~m}^{2}$ ). To compare the differences in the $C_{d}$ of the three meshes intuitively, the train is divided into 156 slices along the train body and each slice is $0.5 \mathrm{~m}$ in length. The time-averaged drag coefficients of the slices are shown in Figure 7.

Table 2. Comparisons of the $C_{d}$ obtained from the numerical and experimental results.

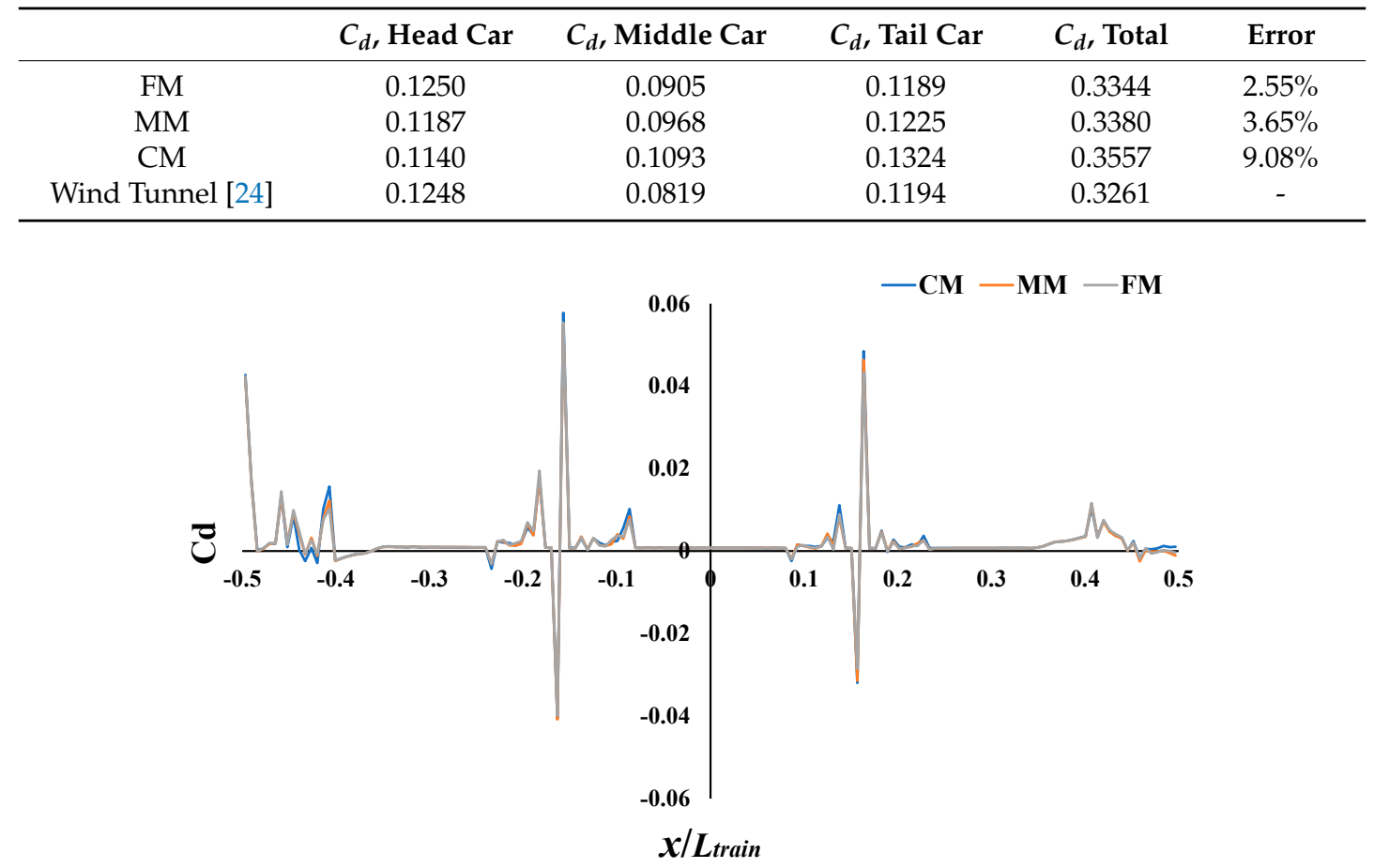

Figure 7. Drag coefficients of the slices of the train.

The results in Table 2 show that the relative error between the FM and wind tunnel test result is only $2.55 \%$, whereas it is $3.65 \%$ for the MM. The errors are relatively small, indicating that both meshes can be used. However, the relative error between the $\mathrm{CM}$ and the wind tunnel test result is larger at $9.08 \%$ and it is not recommended to use the CM. In addition, it can be clearly seen in Figure 7 that the differences in the drag coefficients between the FM and MM are small and their curves are nearly the same. The results of the CM exhibit large deviations from those of the other meshes in the bogies, inter-carriage gaps, and the nose of the trailing car. In general, the MM is suitable for the current simulations with high accuracy and efficiency and it is, therefore, used for the subsequent simulations.

\subsection{Validation of $D M D$}

The two-dimensional cylinder flow is often used as a standard verification example for unsteady flows. In this section, we use the cylinder flow with a Reynolds number of $\operatorname{Re}=8000$ as an example to demonstrate the applicability and accuracy of the DMD method for extracting the coherent flow structures. It has been proved that when $\operatorname{Re}$ is in the subcritical region $\left(300 \leq \operatorname{Re} \leq 3 \times 10^{5}\right)$, the dimensionless frequency of oscillation of the lift force is about St $=0.2$ [25]. Here, St is the Strouhal number and is defined as:

$$
\mathrm{St}=\frac{f \cdot D}{V}
$$


where $D$ is the cylinder diameter, $V$ is the freestream velocity, and $f$ is the physical frequency.

The time history curve of the lift coefficient of the cylinder as obtained by the current numerical simulation is shown in Figure 8. Its corresponding fast Fourier transform (FFT) result is shown in Figure 9; the corresponding dimensionless frequency is $\mathrm{St}=0.201$.

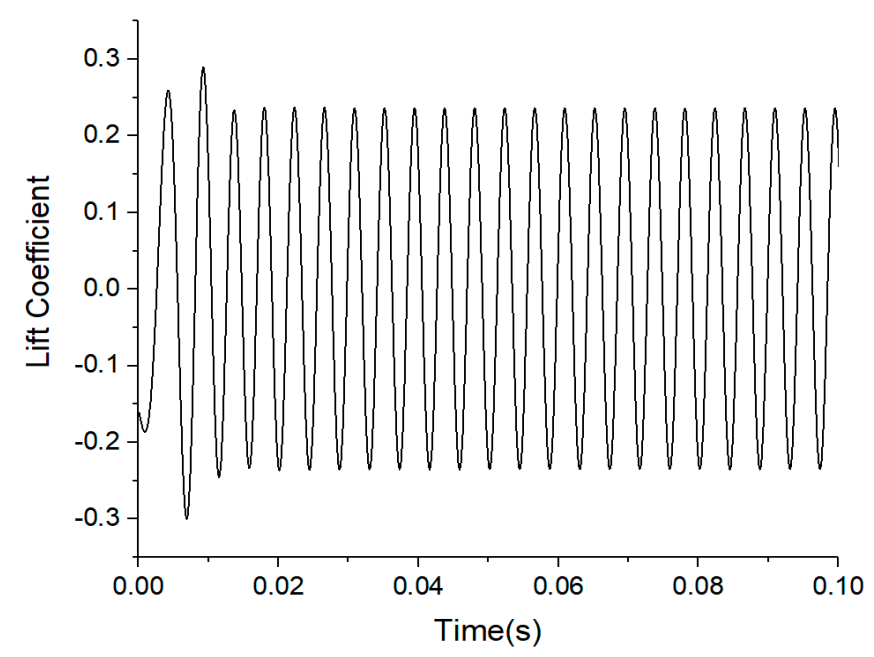

Figure 8. Time history curve of the cylinder lift coefficient, $\operatorname{Re}=8000$.

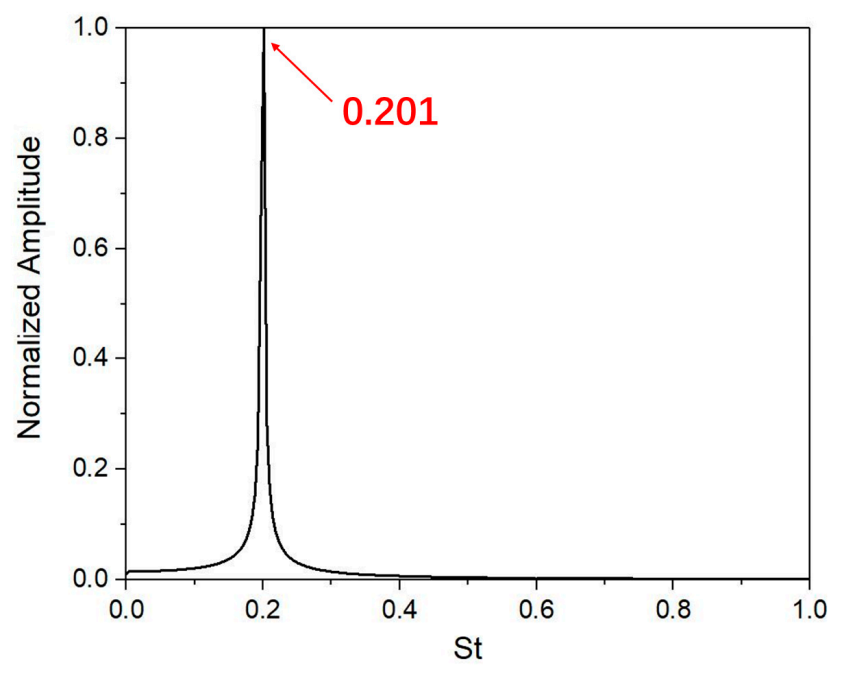

Figure 9. Frequency determined by FFT.

In the DMD analysis, a total of 2343 measurement points in 33 rows and 71 columns are uniformly arranged in an area of $10 D \times 5 D$ just behind the cylinder. The data of the velocity components $u$ and $v$ at 2000 time instants at these points are extracted, resulting in 2000 snapshots of the flow field. Subsequently, 1999 modes along with their corresponding frequencies are obtained using the DMD method. Some selected modes are shown in Figure 10. It is evident that the DMD mode 2 represents the vortex shedding and its dimensionless frequency is $\mathrm{St}=0.199$ (Figure 11), which agrees well with the result obtained by the FFT analysis $(\mathrm{St}=0.201)$. Moreover, the structures of the DMD modes (Figure 10) are consistent with those experimentally obtained by Zhang et al. [26]. These results demonstrate that the DMD method is capable of extracting the coherent flow structures from an unsteady wake flow field. 


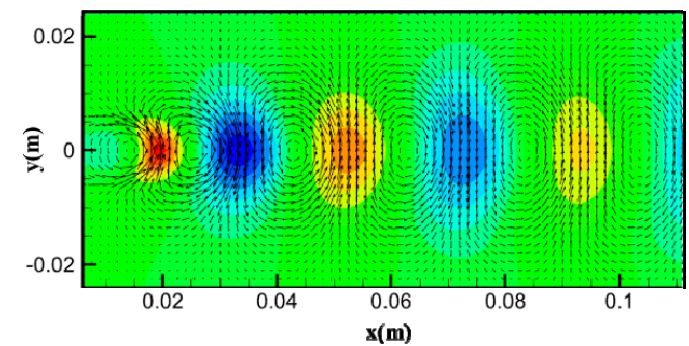

(a) Mode 2

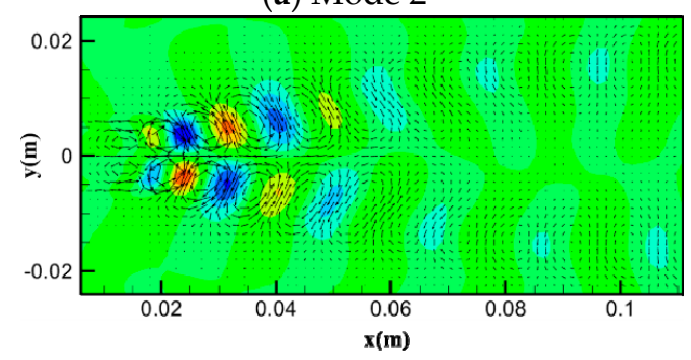

(c) Mode 4

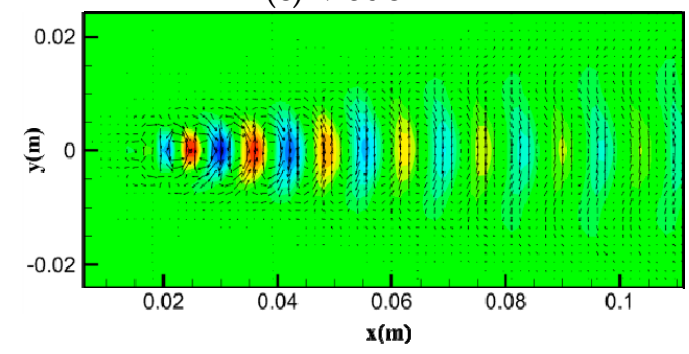

(e) Mode 6

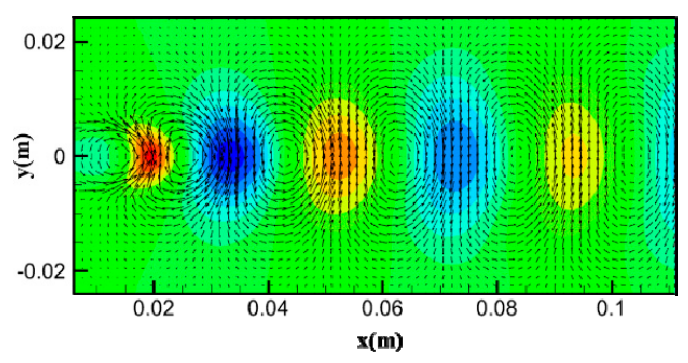

(b) Mode 3

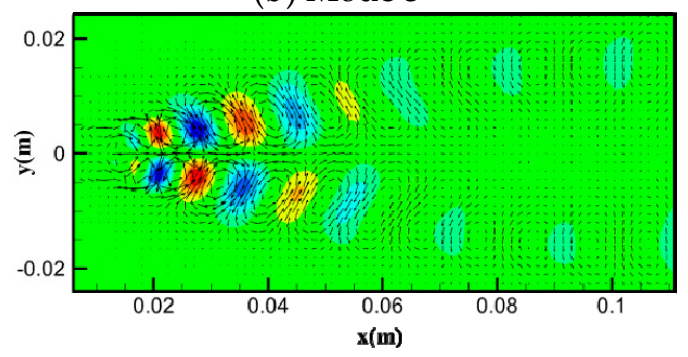

(d) Mode 5

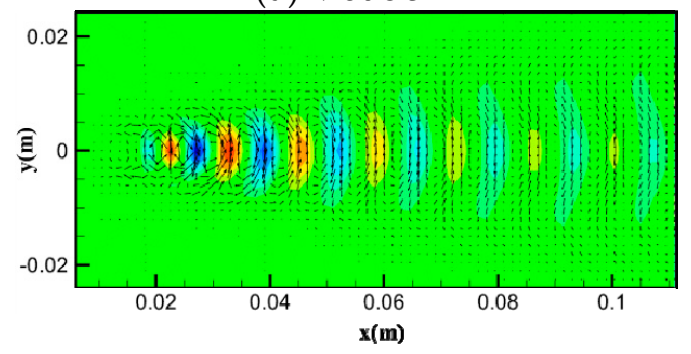

(f) Mode 7

Figure 10. The first six DMD modes of the wake flow of a cylinder.

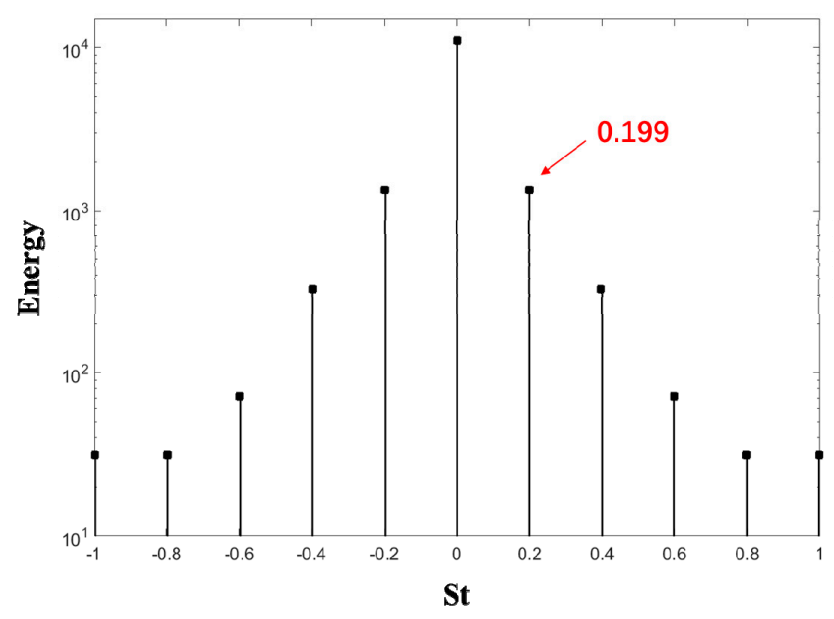

Figure 11. Frequency determined by DMD.

\section{Results and Discussions}

\subsection{Aerodynamic Forces}

In this section, comparisons of the aerodynamic forces of the three high-speed train models are conducted to determine the effects of the bogies on the external flow of the high-speed trains. In the current simulations, the flows are fully developed after $1.0 \mathrm{~s}$ and, thereafter, the aerodynamic forces acting on the train fluctuate around the mean values. Therefore, the averages of the next 5000 time 
steps are taken to count the time-averaged aerodynamic forces of the three models and the drag coefficients; the results are shown in Table 3. The comparisons of the results of the real model and the simplified model, as well as the results of the trailing car for the semi-simplified model and the simplified model show that the drag forces from the bogies are as large as those from the other parts of the train. That is, in the real model, the drag forces from the six bogies contribute approximately $50 \%$ to the total drag forces of the train. As it is well known, the drag forces of the train result from the external flow. The bogies are located at the bottom of the train body where the flow is very complex. These bogies protrude from the smooth bottom of the train and are affected by the incoming flow, which results in unavoidable large drag forces.

Table 3. Time-averaged drag coefficient of the three high-speed train models.

\begin{tabular}{ccccc}
\hline & $C_{d}$, Head Car & $C_{d}$, Middle Car & $C_{d}$, Trailing Car & $C_{d}$, Total \\
\hline Real Model & 0.1187 & 0.0968 & 0.1225 & 0.3380 \\
Semi-simplified Model & 0.0661 & 0.0468 & 0.1088 & 0.2217 \\
Simplified Model & 0.0651 & 0.0468 & 0.0585 & 0.1704 \\
\hline
\end{tabular}

Apart from the average forces of the high-speed train, the existence of the bogies also has a large effect on the oscillation of the aerodynamic forces acting on the train. The time histories of the aerodynamic coefficients of the trailing cars of the three train models are shown in Figure 12. The blue line represents the real model, the orange line represents the semi-simplified model, and the green line represents the simplified model. It can be seen that the aerodynamic forces acting on the trailing car of all the three models oscillate around the average values. However, the amplitudes of the oscillations are quite different. The oscillations of the aerodynamic forces of the simplified model are rather weak compared to the strong oscillations in the real and semi-simplified models. Moreover, the oscillation amplitudes of the forces are larger in the real model than in the semi-simplified model. These differences can be further confirmed by the standard deviations of the aerodynamic coefficients, as shown in Table 4. Obviously, these differences are the result of the different configurations of the bogies in the train, which leads to the conclusion that the existence of the bogies in the high-speed train not only affects the average aerodynamic forces, but also introduces great disturbances to the flow field and consequently results in strong unsteady characteristics in the flow field.

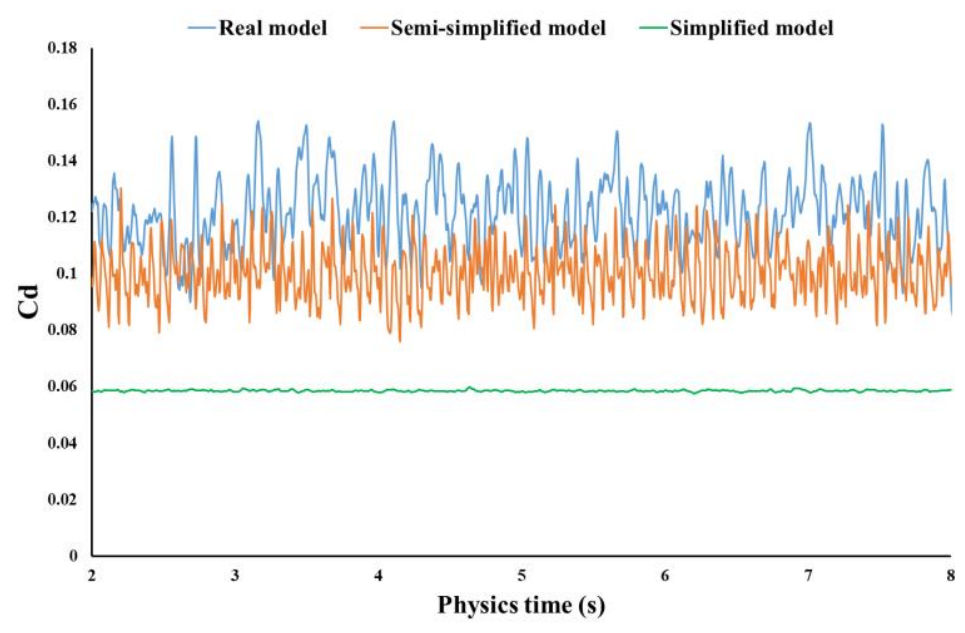

(a) Drag coefficient.

Figure 12. Cont. 


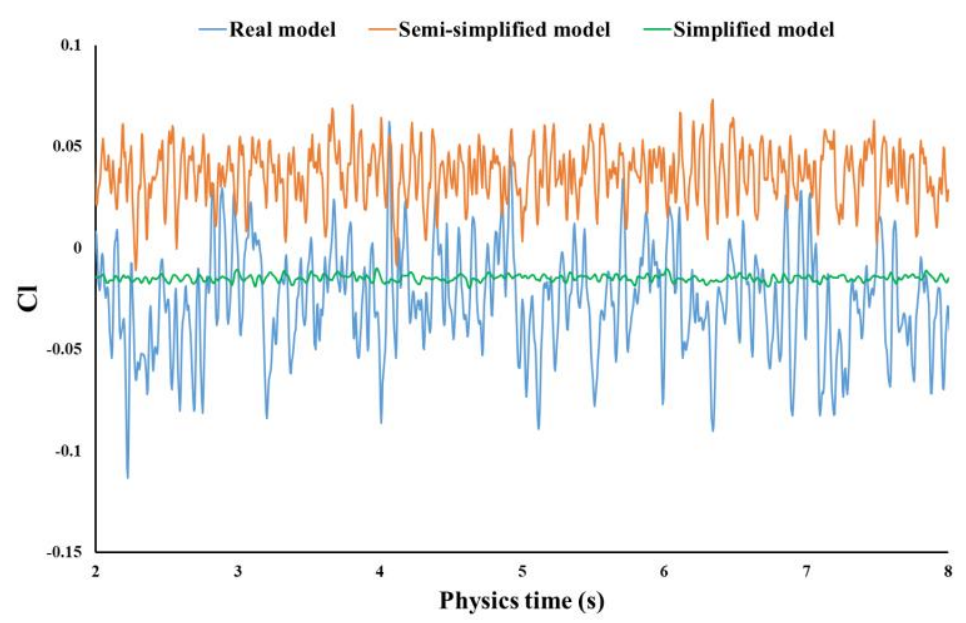

(b) Lift force coefficient.

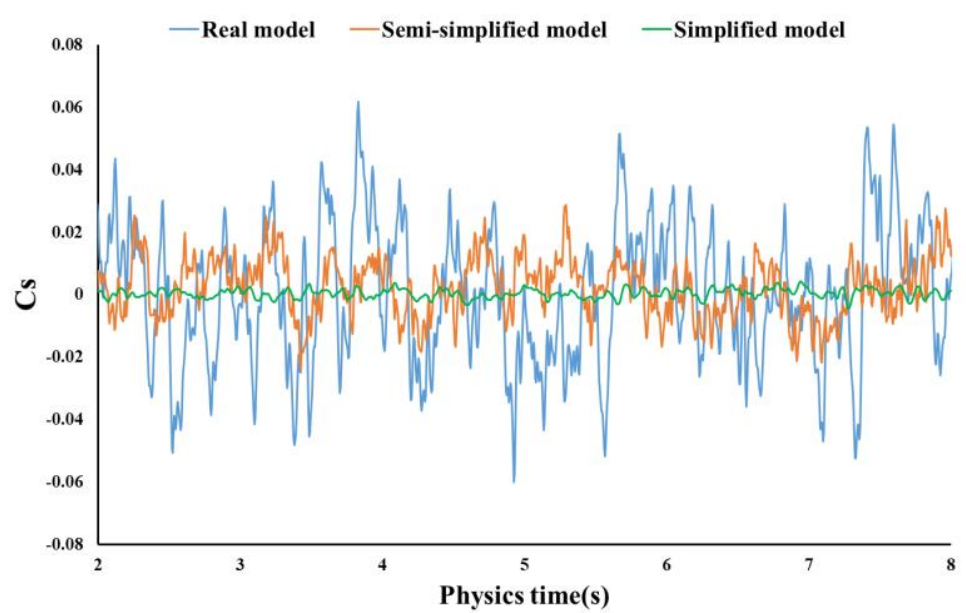

(c) Side force coefficient.

Figure 12. Time history curves of the aerodynamic coefficients of the trailing cars of three models.

Table 4. Standard deviations of the aerodynamic forces of the trailing car of the three high-speed train models.

\begin{tabular}{cccc}
\hline & $\begin{array}{c}\text { Standard Deviation of } \\
\text { Drag Coefficient }\end{array}$ & $\begin{array}{c}\text { Standard Deviation of } \\
\text { Lift Coefficient }\end{array}$ & $\begin{array}{c}\text { Standard Deviation of } \\
\text { Side Force Coefficient }\end{array}$ \\
\hline Real Model & $1.19 \times 10^{-2}$ & $2.57 \times 10^{-2}$ & $2.22 \times 10^{-2}$ \\
Semi-simplified Model & $0.93 \times 10^{-2}$ & $1.35 \times 10^{-2}$ & $0.94 \times 10^{-2}$ \\
Simplified Model & $0.03 \times 10^{-2}$ & $0.15 \times 10^{-2}$ & $0.15 \times 10^{-2}$ \\
\hline
\end{tabular}

\subsection{Time-Averaged Flow Field}

\subsubsection{Slipstream}

The train is kept stationary in the current simulations and the velocities of the freestream and moving ground are set equal to the train running speed $(V=300 \mathrm{~km} / \mathrm{h})$ but in opposite directions. Therefore, when analyzing the slipstream, the stationary ground should be used as the reference frame and the slipstream velocity is defined as:

$$
u_{w}=\sqrt{(u-V)^{2}+v^{2}+w^{2}},
$$


where $u, v$, and $w$ are the time-averaged velocity components in the streamwise, spanwise, and vertical directions.

The distributions of the slipstream velocity at $(y, z)=(3,0.2) \mathrm{m}$ and $(3,1.4) \mathrm{m}$ are shown in Figure 13. The position of $z=0.2 \mathrm{~m}$ is very close to the upper surface of the rail and is greatly influenced by the underbody flow and ground effect. The position of $z=1.4 \mathrm{~m}$ is about half the train height and is also at the same level as the height of a human body; this is important with regard to safety. As shown in Figure 13, the slipstream velocity peaks occur at positions corresponding to the noses of the head and trailing cars. This occurs because when the train moves forward, the airflow in front of the nose of the head car is pushed forward and sideward. The airflow changes dramatically in the streamlined region of the head car where the cross-sectional area exhibits significant changes; this results in the velocity peak of the slipstream. The slipstream distributions of the three train models are similar near the noses of the head cars. Similarly, the cross-sectional area in the streamlined region of the trailing car varies and, therefore, a significant change occurs in the airflow, resulting in another velocity peak of the slipstream. Moreover, the slipstream near the nose of the trailing car is also affected by separation and recirculation regions and shedding vortices.

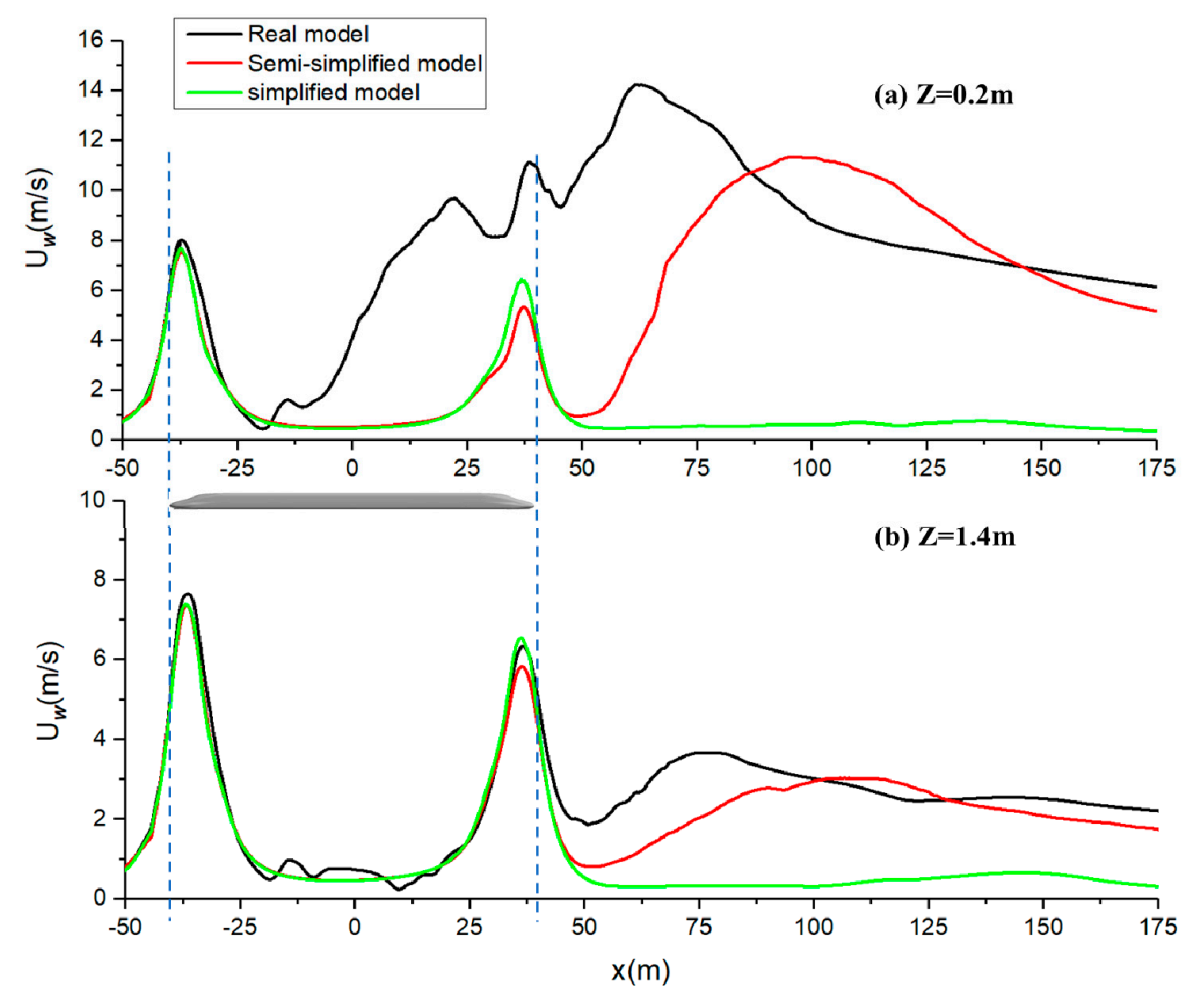

Figure 13. Distributions of the slipstream velocity of the three train models at $y=3 \mathrm{~m}$ and (a) $\mathrm{z}=0.2 \mathrm{~m}$ and $(\mathbf{b}) \mathrm{z}=1.4 \mathrm{~m}$.

A comparison of the three curves in Figure 13a shows that the airflows change little in the middle of the simplified and semi-simplified train models due to their constant cross-sectional shapes, which results in an approximately zero slipstream velocity. However, in the real model, a strong slipstream occurs in the middle of the train and becomes larger at the end of and far away from the train. This result is in agreement with the research results of Hemida et al. [13,14]. The differences between the real model and the simplified and semi-simplified models are the different configurations of the bogie in the middle of the train models. The bogie has a significant influence on the external flow field of the train and increases the nearby flow and hence the slipstream. At $z=1.4 \mathrm{~m}$, as shown in Figure 13b, the effect of the bogie on the flow field is smaller, which results in the similar distributions 
of the slipstream velocity (close to zero) in the middle of the three models. It can be concluded that bogies have large effects on the flow field of high-speed trains, especially on the underbody flow field.

Since the maximum peak of the slipstream velocity occurs in the wake of the real model (Figure 13a), it motivates us to pay more attention to the effects of bogies on the slipstream in the wake. As shown in Figure 13, similar slipstream velocity values are observed in the wake of the semi-simplified and real train models and the values are much larger than those of the wake of the simplified model, which are approximately zero. Moreover, the peak position in the real model is closer to the trailing car than in the semi-simplified model; in contrast, no obvious slipstream velocity can be observed in the wake of the simplified model. Again, these differences can be attributed to the different configuration of the bogies in the models. It can be concluded that the existence of the bogie not only has a strong interference effect on the nearby flow field, but also greatly affects the flow field around the trailing car and the wake, thereby increasing the wake of the high-speed train.

\subsubsection{Turbulent Kinetic Energy}

The TKE defined by Equation (16) is one of the most important variables used in the evaluation of the intensity of the turbulence in turbulent flow and it is directly related to the transport of momentum in the flow:

$$
T_{E}=\frac{1}{2}\left(\overline{u^{\prime} u^{\prime}}+\overline{v^{\prime} v^{\prime}}+\overline{w^{\prime} w^{\prime}}\right)
$$

where $u^{\prime}, v^{\prime}$, and $w^{\prime}$ are the fluctuation velocity components in the streamwise, spanwise, and vertical directions, respectively; the symbol "-" indicates the time average of the corresponding variables. The streamwise distributions of the time-averaged TKE at different locations of the wake of the three models are shown in Figures 14 and 15.
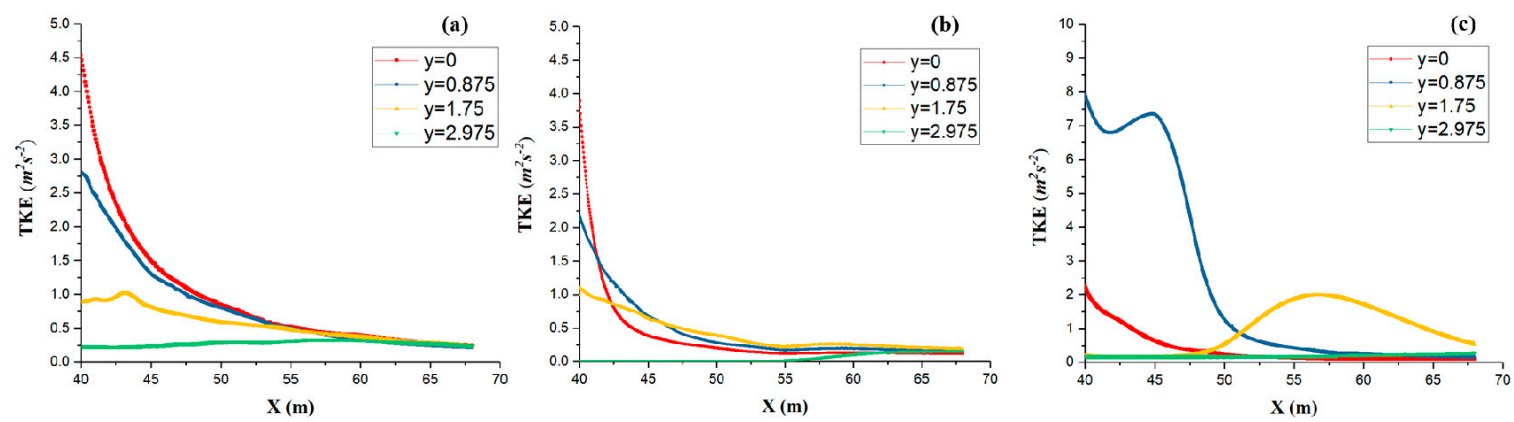

Figure 14. Streamwise distributions of time-averaged TKE at different spanwise positions $(\mathrm{z}=0.2 \mathrm{~m})$ : (a) real model, (b) semi-simplified model, and (c) simplified model.
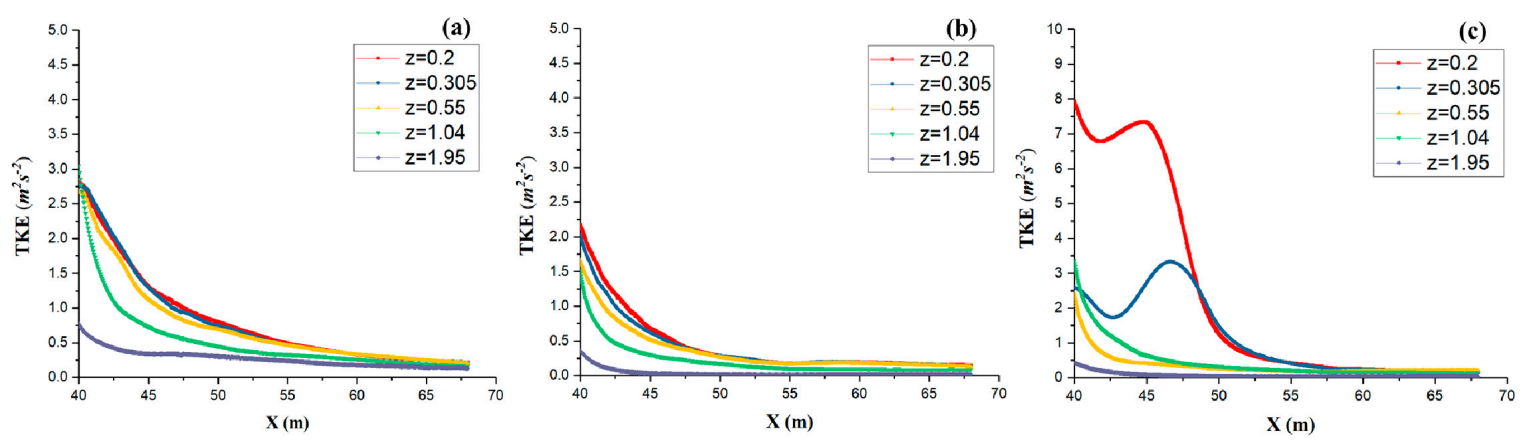

Figure 15. Streamwise distributions of time-averaged TKE at different vertical positions $(\mathrm{y}=0.875 \mathrm{~m})$ :

(a) real model, (b) semi-simplified model, and (c) simplified model.

A comparison of the streamwise TKE distributions in the wake of the three models at different span positions $(y=0,0.875,1.75$, and $2.975 \mathrm{~m}$ ) (Figure 14) indicates that the TKE distributions are 
similar for the real and semi-simplified models. The TKE is large near the nose of the trailing car and at the spanwise middle of the wake $(y=0$ and $0.875 \mathrm{~m})$ and it monotonously decreases downstream of these two streamwise positions. At the position of $y=2.975 \mathrm{~m}$, which is the outer side in the spanwise direction, TKE rises slightly and then gradually attenuates.

However, the TKE distributions in the wake are quite different for the simplified model. The TKE at $y=0$ is relatively small, whereas it is larger at $y=0.875 \mathrm{~m}$. After the peak at $x=45 \mathrm{~m}$, the TKE decreases monotonously downstream. For $y=1.75 \mathrm{~m}$, which is the outer side in the spanwise direction, the TKE is small near the nose of the trailing car, then exhibits a significant increase, reaches a peak at $x=57 \mathrm{~m}$, and finally gradually decreases. For $y=2.975 \mathrm{~m}$, the TKE is almost zero. The TKE distributions at different spanwise positions are closely related to the movements of the vortex structures in the wake, and hence implies information about positions and incidences of vortices.

The streamwise distributions of the TKE in the wake of the three models at different heights $(z=0.2,0.305,0.55,1.04$, and $1.95 \mathrm{~m})$ are shown in Figure 15. It is observed that the TKE distributions in the wake are similar for the real and semi-simplified models. The largest TKE values occur near the nose of the trailing car and then the values monotonously decrease downstream. As the height increases, the TKE values decrease. However, the TKE distributions in the wake are different for the simplified model. At the positions of $z=0.2$ and $0.305 \mathrm{~m}$, except for the TKE peaks near the nose of the trailing car, another distinct peak is observed in the range of $45 \mathrm{~m}<x<50 \mathrm{~m}$. In addition, the TKE values no longer decrease as the height increases in the simplified model because the TKE at $z=1.04 \mathrm{~m}$ is larger than that at $z=0.55 \mathrm{~m}$, whereas it is close to zero at $z=1.95 \mathrm{~m}$.

\subsection{Instantaneous Wake Flow}

In this section, the effects of the bogies on the generation and the evolution of the flow structures of the wake vortices are analyzed by comparing the pressure, velocity, and vorticity distributions of the three models. The instantaneous structures of the wake vortex of the three models are shown in Figure 16 as iso-surfaces of the second invariant of the velocity gradient $Q$, which is defined as:

$$
Q=-\frac{1}{2} \frac{\partial u_{i}}{\partial x_{j}} \frac{\partial u_{j}}{\partial x_{i}}
$$

In Figure 16a, a pair of large streamwise vortices characterized by alternating generation and shedding is observed in the wake of the real model; these vortices have a large influence range in the spanwise direction. Not coincidentally, a pair of slightly smaller streamwise vortices also appears in the semi-simplified model wake but with slightly weaker alternating generation and shedding and a slightly smaller influence range, as shown in Figure 16b. However, as shown in Figure 16c, the wake of the simplified model is clearly characterized by two layers of vortex structures, which are quite different from those in the other two models and result in the two TKE peaks in the vertical direction; the influence range in the spanwise direction is rather small in the wake of the simplified model (Figure 15). A comparison of the structures of the wake vortex of the three models for the same $Q$ value demonstrates that the wake vortices are larger in the real model than the semi-simplified model and both are significantly larger those in the simplified model. However, due to the small size of the wake vortex in the simplified model, the exact differences cannot be easily determined and further analysis is required.

It is also observed in Figure 16c that the lower pair of vortices in the simplified model originates from the cowcatcher, whereas the upper pair of vortices originates from the nose of the trailing car; this is illustrated in more detail in Figure 17. At $x=37 \mathrm{~m}$, a pair of internally rotating vortices (A and $A^{\prime}$ ) occurs on both edges of the cowcatcher. As shown in Figure 18, the pressure on the cowcatcher is lower than the pressure on the bottom of the trailing car, which results in the air flowing over the edge of the cowcatcher L1 (as shown in Figure 19) from the bottom of the trailing car due to pressure gradients. This vortex generation mechanism is quite similar to that of wing-tip vortices in airplanes. Then, at $x=38 \mathrm{~m}$, the size and strength of the lower pair of vortices gradually increase. 
At $x=38.9 \mathrm{~m}$, the other two pairs of vortices (B-B' and $\mathrm{C}^{\left.-\mathrm{C}^{\prime}\right)}$ begin to detach from the nose of the trailing car. The rotating directions of these two pairs of vortices are opposite to each other. The detailed generating mechanism of these two pairs of vortices has been described in several studies $[6,9,27]$. The downstream airflow at the end of the trailing car can be divided into three parts: the flow on the top of the streamlined surface, the flow on the lateral surfaces, and the flow beneath the streamlined surface. The flow velocity on the top of the streamlined surface decreases rapidly, whereas the flows on the lateral surfaces maintain their velocity, resulting in strong velocity shear layers and formations of vortex structures. One difference between the model simulated in this study and those in previous studies is that the cross-section of the nose is elliptical and the nose geometry is transversely zygomorphic with a small curvature, as shown in Figure 19; this configuration results in the same velocity gradients on L2 and L3, induces rotating airflow, and ultimately generates two pairs of vortices. However, the strength of the upper pairs of vortices decreases rapidly. At $x=44.5 \mathrm{~m}$, the vortex pair $C$ and $\mathrm{C}^{\prime}$ disappears and, at $x=55 \mathrm{~m}$, another upper pair of vortices (B and $\left.\mathrm{B}^{\prime}\right)$ disappears, whereas the lower pair of vortices is still clearly visible but the vortices are spaced further apart.
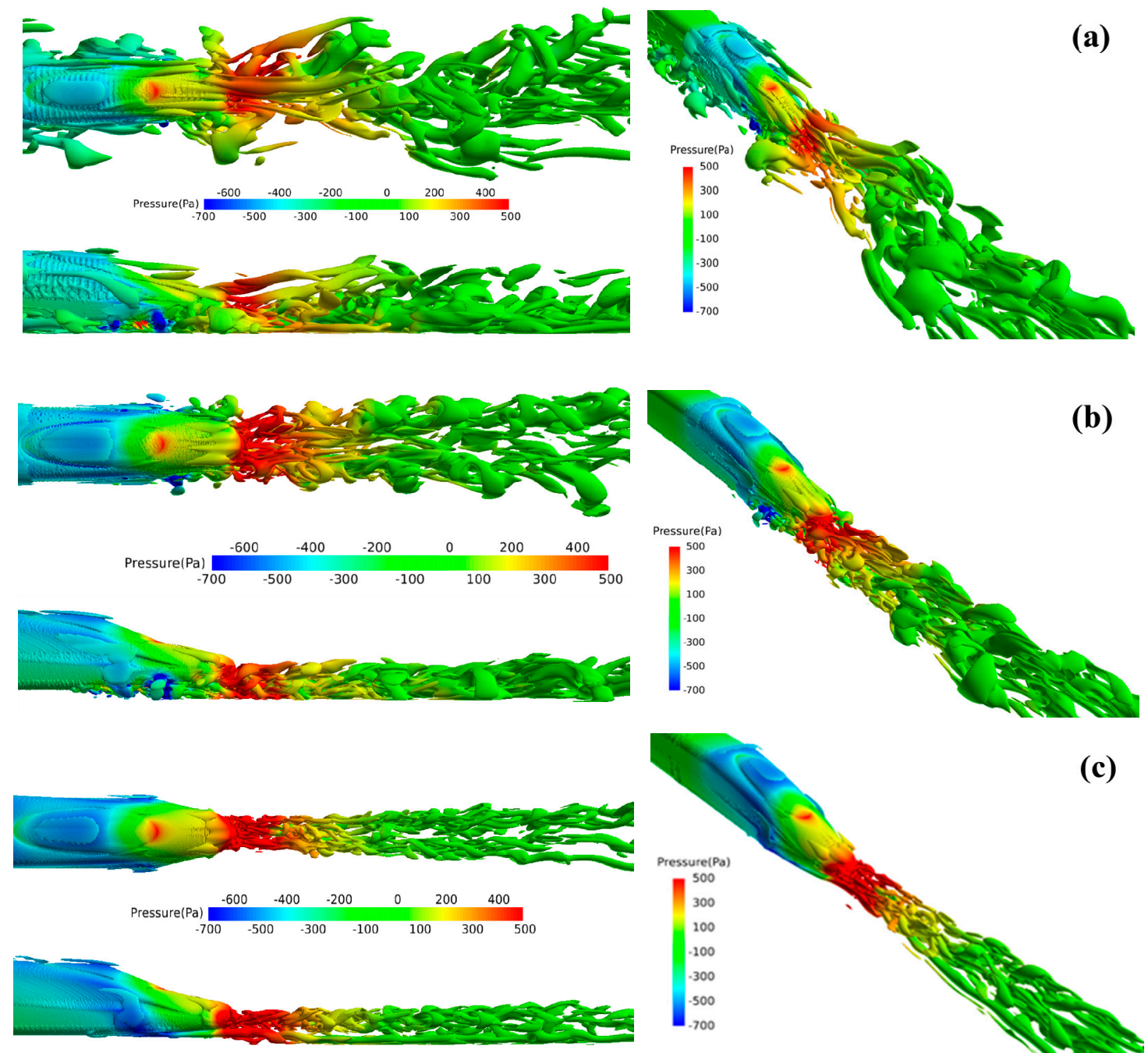

(c)

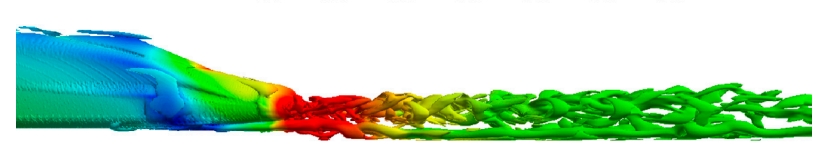

Figure 16. Iso-surfaces of $Q(Q=200, t=5 \mathrm{~s})$ of the (a) real model, (b) semi-simplified model, and (c) simplified model. 

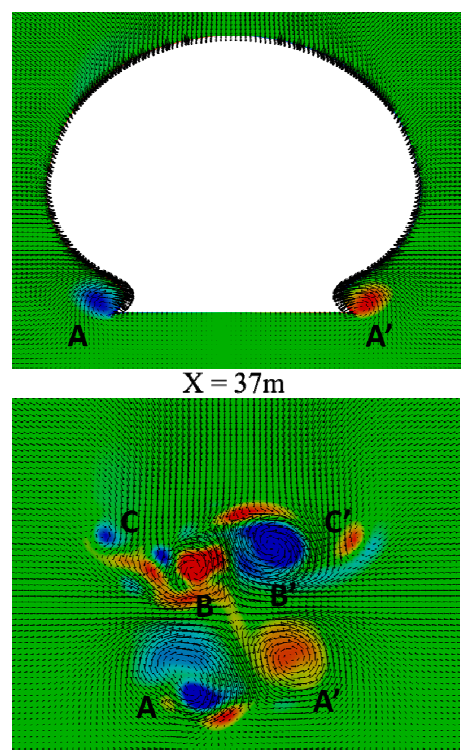

$X=39.5 m$

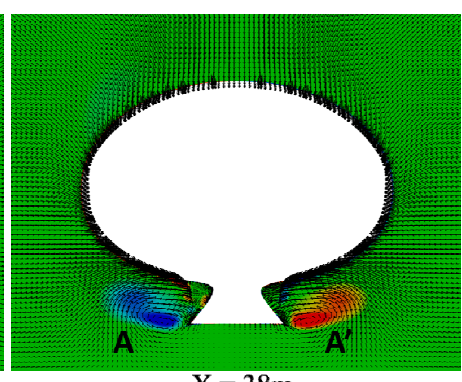

$X=38 m$

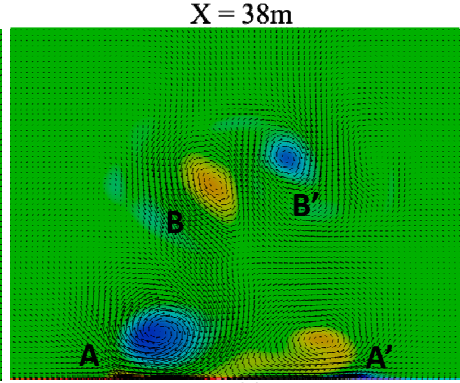

$\mathrm{X}=44.5 \mathrm{~m}$

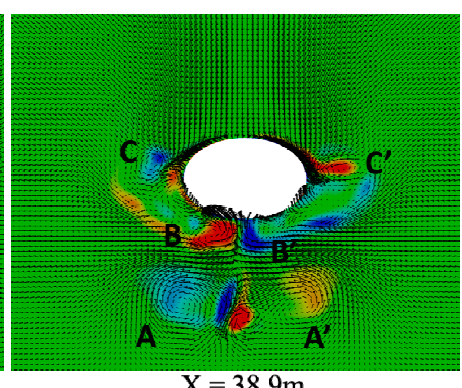

$\mathrm{X}=38.9 \mathrm{~m}$

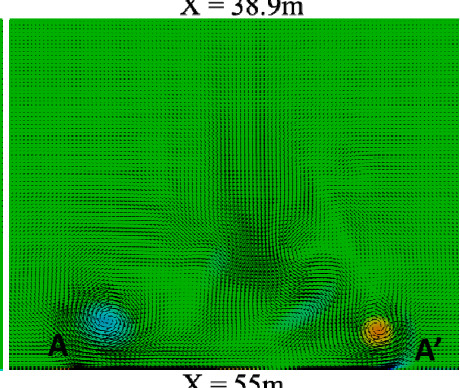

Figure 17. Contours of the instantaneous streamwise vorticity of the simplified model at different cross-sections.

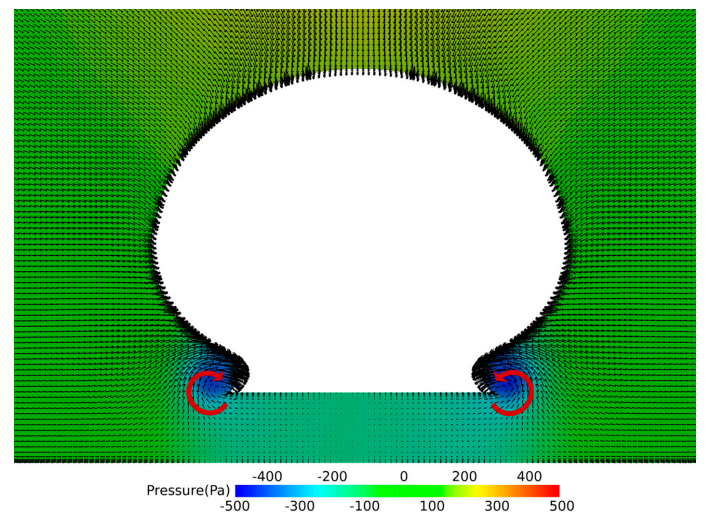

Figure 18. Pressure contour of the simplified model at $x=37 \mathrm{~m}$.

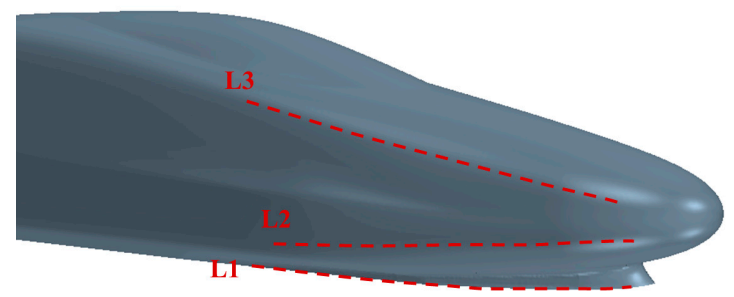

Figure 19. Geometry of the nose of the trailing car in the simplified model.

Numerous studies [1,5-9] have demonstrated that a pair of large counter-rotating streamwise vortices exists in the wake and this is also clearly observed in Figure 20 in the real model. These vortices move towards the ground and away from each other, accompanied by alternating vortex shedding, which is a result of their mutual inductions and interactions with the ground. The spatial structure of these wake vortices is quite complex and looks like a "branch". At $x=37 \mathrm{~m}$, a clockwise vortex A detaches itself from the left side. At $x=43 \mathrm{~m}$, the intensity of the previously detached vortex $\mathrm{A}^{\prime}$ on the right side reaches its maximum and becomes dominant. At $x=47.5 \mathrm{~m}$, the intensity of vortex A on the left side reaches its maximum and becomes dominant, while vortex $\mathrm{A}^{\prime}$ nearly disappears at this location. At $x=56 \mathrm{~m}$, the anticlockwise vortex $\mathrm{A}^{\prime}$ on the right side is dominant again. The size, intensity, and spacing of the wake vortices are larger for the real model than the simplified model. 
Clearly, the existence of the bogies has a large interference effect on the wake flow and results in large differences in the intensity between the two vortices in the same pair. In order to better demonstrate this point, the vorticity contours and velocity vectors of the semi-simplified model at different cross-sections are shown in Figure 21. It is observed that in the wake of the semi-simplified model, only one pair of vortices (A and $\left.A^{\prime}\right)$ exists and the vortices alternately dominate downstream.

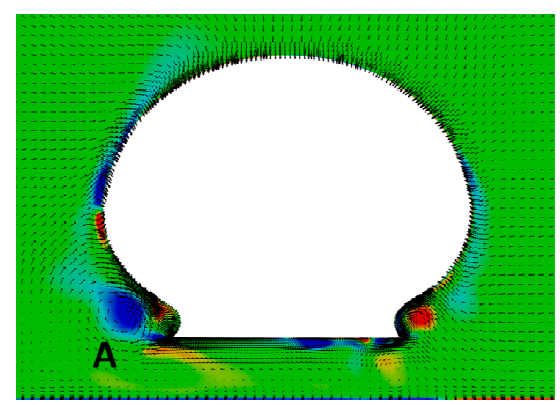

$$
\mathrm{X}=37 \mathrm{~m}
$$

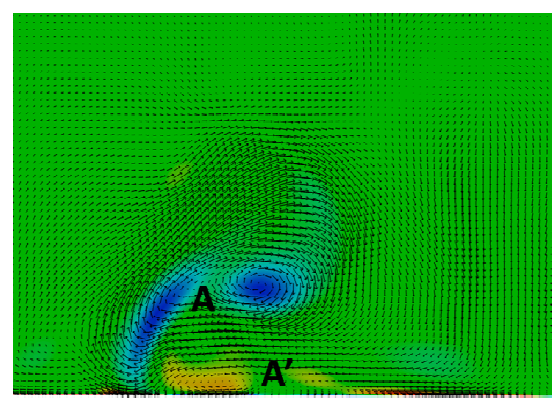

$\mathrm{X}=47.5 \mathrm{~m}$

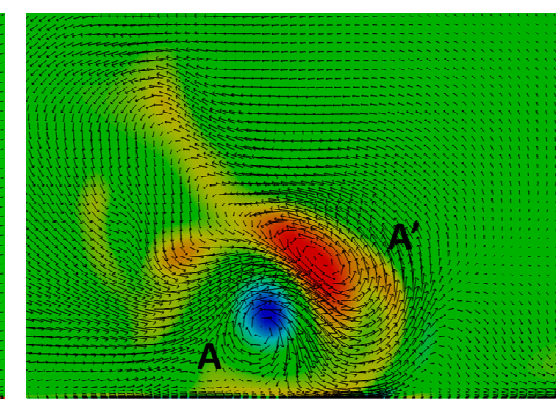

$X=43 m$

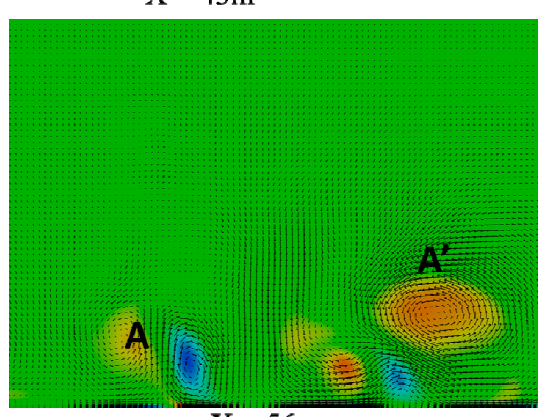

$\mathrm{X}=56 \mathrm{~m}$

Figure 20. Contours of the instantaneous streamwise vorticity of the real model at different cross-sections.

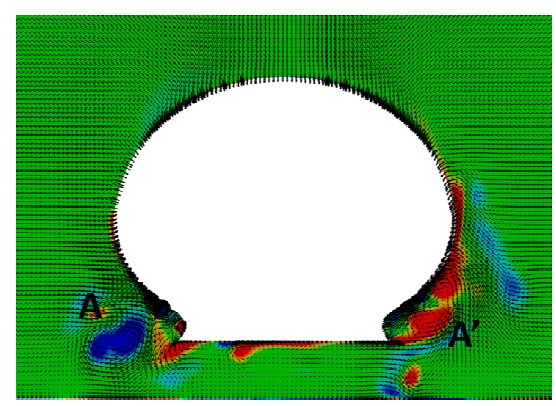

$\mathrm{X}=37 \mathrm{~m}$

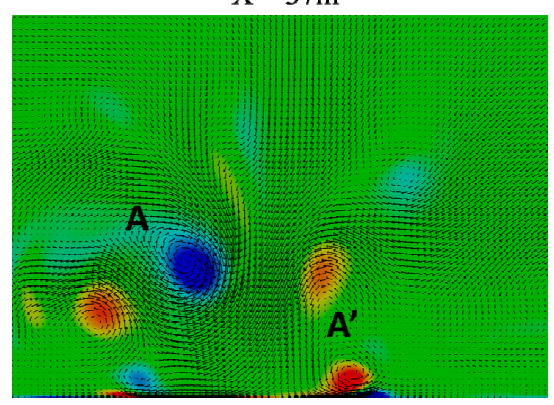

$X=43 m$

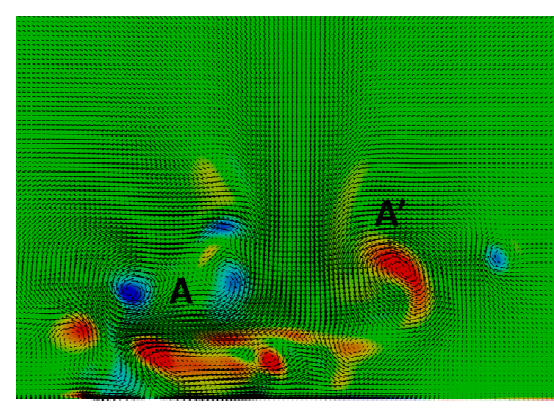

$X=41 m$

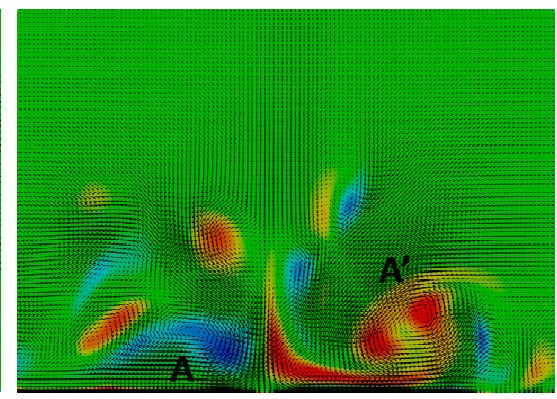

$\mathrm{X}=47 \mathrm{~m}$

Figure 21. Contours of the instantaneous streamwise vorticity of the semi-simplified model at different cross-sections.

These results indicate the similarity in the wake flows of the real model and semi-simplified model, which are characterized by a pair of counter-rotating streamwise vortices that alternately dominate. However, the wake flow of the simplified model is quite different and is characterized by 
two layers of vortex structures. The upper pairs of vortices decay rapidly, whereas the lower pair of vortices develops over a longer distance. The size, influence range, and other characteristics of these two layers of wake vortices are also quite different. The similarity of the wake flow between the real model and the semi-simplified model is due to the similar configurations of the bogies. The simplified model has no bogies; therefore, its wake flow is quite different from those of the other two models. Overall, the results lead to the conclusion that the bogies play a significant role in the generation and evolution of the wake vortices of high-speed trains.

\subsection{Results of $D M D$}

In order to determine the characteristics of the wake vortices of the three models accurately and clearly, the DMD method is applied to identify the coherent wake vortex structures and their evolutions. A total of $160 \times 50$ data points at the cross-section of $x / \mathrm{H}=1$ are selected to extract the velocity, pulsating pressure, and vorticity of the flow field to create a snapshot of the flow field. In order to ensure the convergence of the extracted DMD modes, sufficient snapshot samples are needed to obtain sufficient periods of the unsteady flow. Following the convergence verification methods used by Muld et al. [15], it is proved that in this study, a sampling time-step of $\Delta t=0.25 T_{\text {ref }}$ and a total sample time of $T_{\mathrm{A}} \geq 250 T_{\text {ref }}$ provides at least 45 periods and ensures the convergence of the first four DMD modes. $T_{r e f}$ is the reference time and is defined as:

$$
T_{\text {ref }}=\frac{H}{V} \text {. }
$$

In the DMD, the strong unsteady wake flow is decomposed into several modes, which are sorted in descending order based on the mode energy (Equation (9)). Figure 22 shows the energy distributions of the first 100 modes of the real and simplified models. It can be seen that mode 1 is the most important mode with the maximum energy, which is about twice as high as that of mode 2 . The higher order modes exhibit a series of unsteady oscillations in the flow field and their energies decrease successively. The energies of the higher order modes are lower for the simplified model than the real model, which means that the unsteady oscillations are weaker in the wake flow of the simplified model. Therefore, the existence of the bogies is related to the unsteady oscillation characteristics of the wake. In this section, the first two modes with a high energy ratio are analyzed to clearly explain the evolution of the wake vortices.

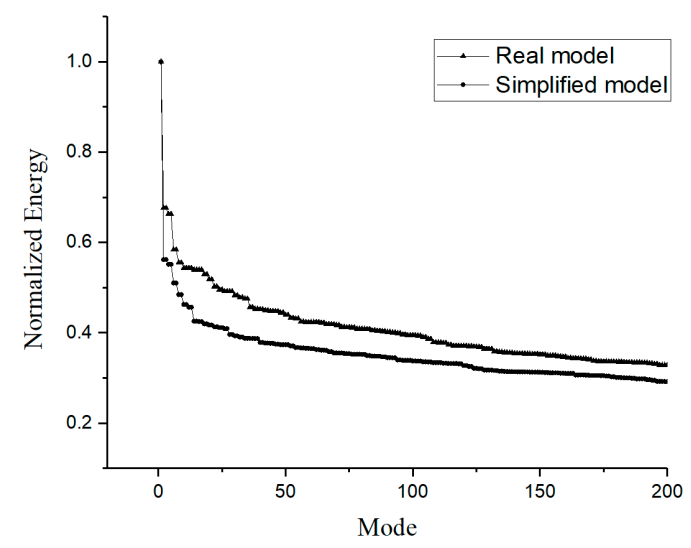

Figure 22. Energy distributions of the DMD modes 1-100 of the real and simplified models.

The flow fields of mode 1 and mode 2 and the corresponding reconstruction at different time instances of the simplified and real model are shown in Figures 23-27, respectively. As demonstrated in Section 5.3, the wake of the simplified model is layered. Therefore, it is divided into two regions in the DMD analysis. The lower layer wake of the simplified model is shown in Figure 23. In the DMD, all modes except mode 1 appear in pairs. Therefore, we only analyze one of each pair of modes 
here. Mode 1 is exactly the mean flow without periodic change, which is one of the properties in this type of flow with dominant mean flow. As shown in Figure 23, Mode 1 shows two symmetrical, counter-rotating streamwise vortices, whereas mode 2 and higher exhibit oscillations around the mean flow. Obvious periodicity can be observed in mode 2. An anticlockwise vortex is formed in phase 1; subsequently, the intensity of this vortex weakens and it ultimately changes into a clockwise vortex in phase 3. When this periodically changing vortex in Mode 2 is added to the pair of counter-rotating vortices in mode 1, it results in an asynchronous and periodic change in the intensities of the two vortices, which is clearly reflected in the DMD reconstructions. Figure 24 shows three typical snapshots of the flow field after DMD reconstruction of the first two modes. The intensity of the right vortex is greater than that of the left vortex in phase 1 and the vortex core is larger as well. In phase 2, the intensities of the two vortices are almost the same. The left vortex dominates in phase 3 ; the intensity of the left vortex is greater and the vortex core is larger.
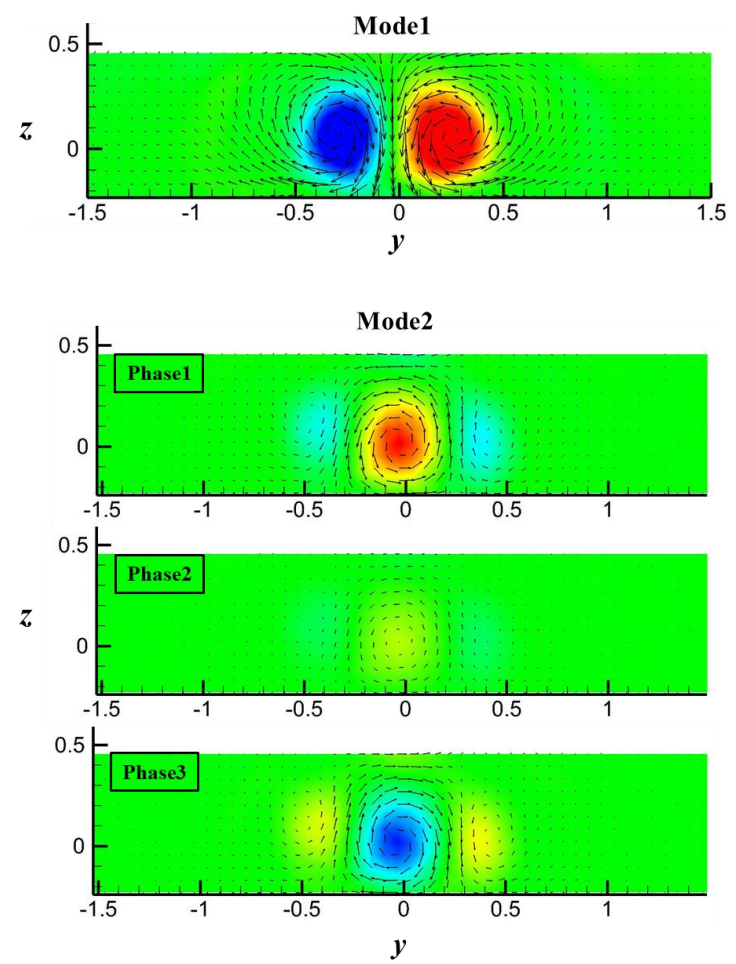

Figure 23. DMD mode 1 and mode 2 of the lower layer wake of the simplified model.

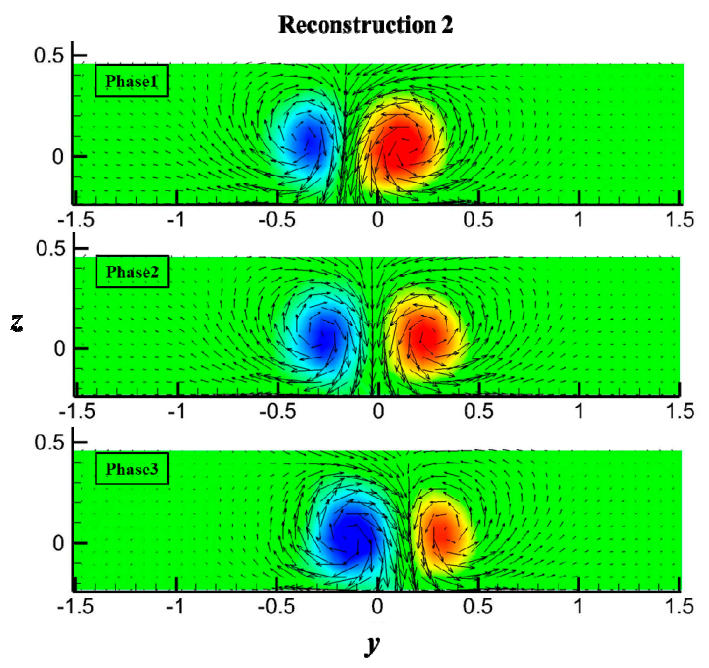

Figure 24. Reconstructed flow fields of the lower layer wake of the simplified model. 
A similar analysis is conducted for the upper wake vortices of the simplified model, as shown in Figure 25. In mode 1, there are two pairs of vortices without periodic changes and each pair consists of two counter-rotating streamwise vortices. Three vortex structures are observed in mode 2 and the intensities and rotation directions of the three vortices change periodically over time from blue-red-blue in phase 1 to red-blue-red in phase 3. Similar to the lower wake vortices, when the three periodically-changing vortex structures in mode 2 are added to the two pairs of vortices in mode 1 , the intensities of the vortices change asynchronously in the reconstructed flow field and the position of the vortex cores may periodically move, indicating alternation of vortex shedding.

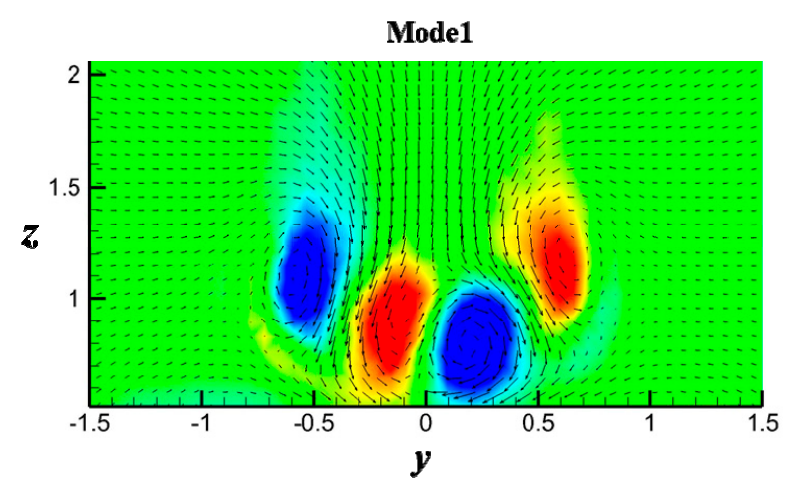

Mode2
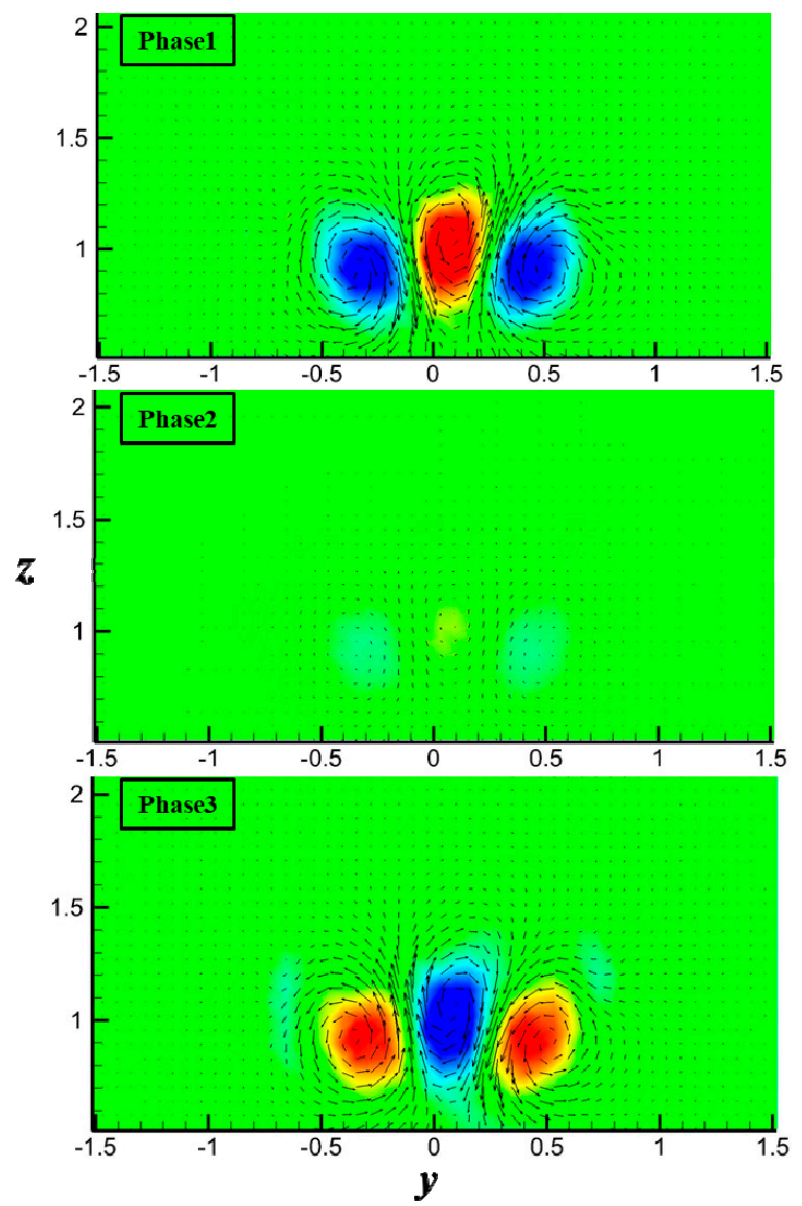

Figure 25. DMD mode 1 and mode 2 of the upper layer wake of the simplified model. 

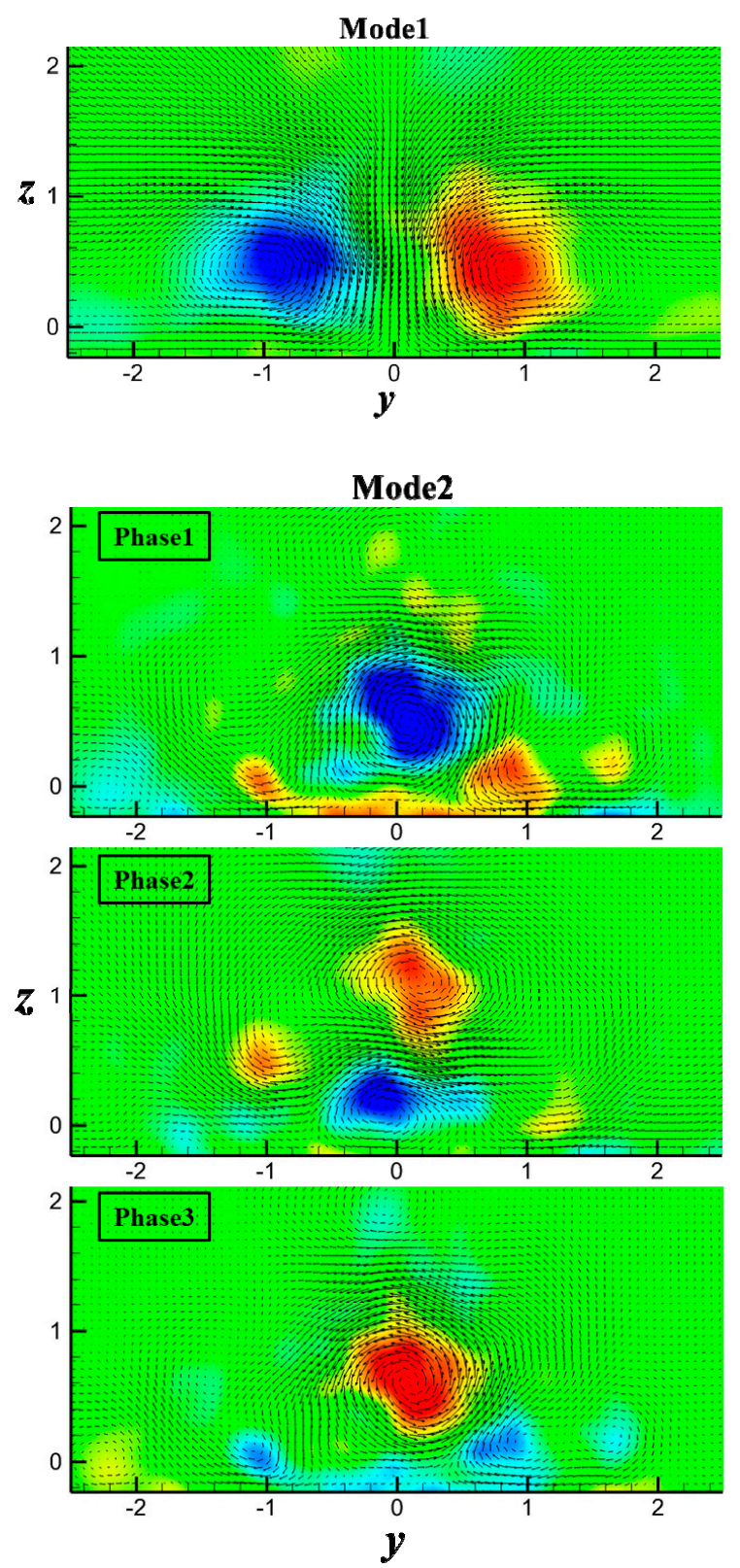

Figure 26. DMD mode 1 and mode 2 of the wake of the real model.

A DMD analysis is also conducted for the wake of the real model and the flow fields of the first two modes and their reconstructions are obtained, as shown in Figures 26 and 27. Mode 1 shows a pair of large, counter-rotating streamwise vortices. Mode 2 consists of one main vortex structure whose rotation direction changes periodically over time from clockwise in phase 1 to anticlockwise in phase 3 . In addition, the vortex structure in mode 2 also changes along the $z$-direction. In Figure 26, as the blue vortex moves downward, a red vortex is generated at the top of the frame and then gradually moves downward into the zone of $0<\mathrm{z}<1$ and becomes the dominant vortex structure. Subsequently, as the red vortex moves downward, a blue vortex is generated at the top of the frame, etc. When this periodically changing vortex structure in mode 2 is added to the counter-rotating pair of vortices in mode 1 , it also results in the asynchronously alternating enhancement or attenuation of the intensity of the two vortices in the reconstructed flow field (Figure 27).

The characteristics of the lower pair of vortices in the simplified model are similar to those in the real model in terms of the coherent structures, rotating directions of the vortices, and other characteristics of the wake. In both models, there is a pair of counter-rotating streamwise vortices, 
whose intensity alternately increases or decreases over time, reflecting the alternating generation and shedding of the wake vortices. The only difference is that the strength and size of the trailing vortices of the real model are larger than those of the lower pair of vortices of the simplified model, along with larger influence on the nearby wake flow.

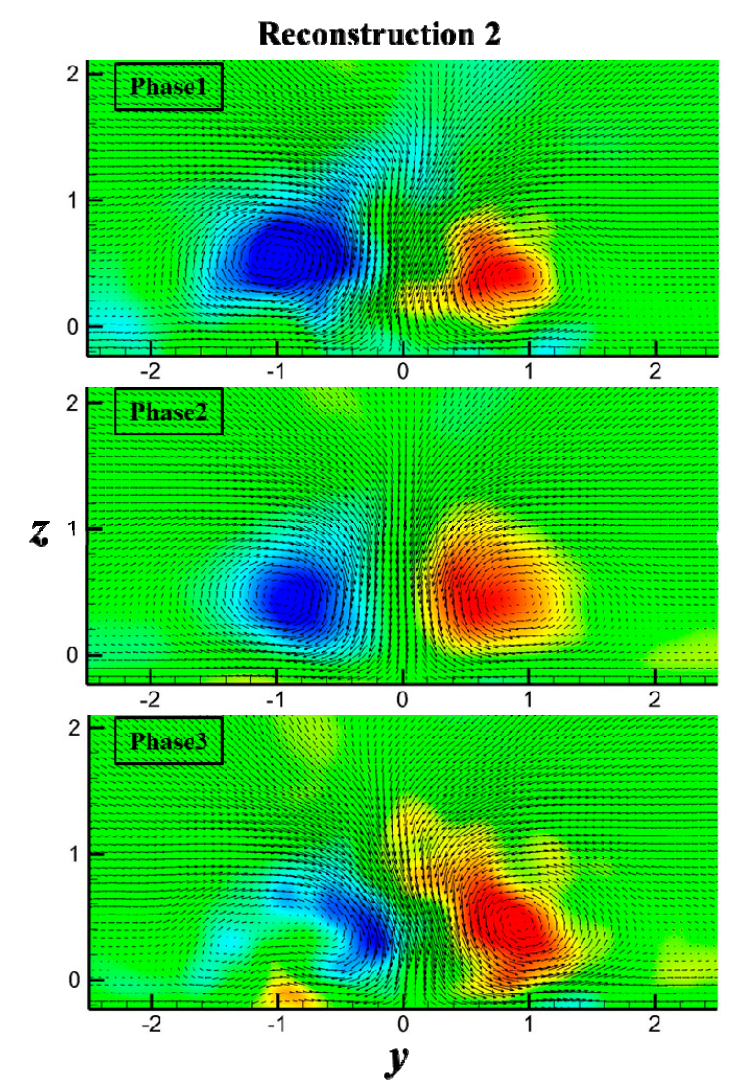

Figure 27. Reconstructed flow fields of the wake of the real model.

\subsection{Mechanism of Generation and Evolution of Trailing Vortices}

Based on above analyses, a mechanism of the generation and evolution of the trailing vortices in a high-speed train is obtained and illustrated as follows. Two sources of vortex generation are inherent in the trailing car of the simplified model and they are the result of the unique geometry of the train. The first source is the cowcatcher of the trailing car; the pressure on the cowcatcher is lower than that on the bottom of the trailing car and the air flows over the edge of the cowcatcher from the bottom of the trailing car due to a pressure gradient, resulting in the formation of a pair of internally rotating vortices near the edge of cowcatcher. This vortex generation mechanism is quite similar to that at the wing tips in airplanes. The other source is the nose of the trailing car due to its unique geometry; the flows on the upper and the lower surfaces of the nose slow down significantly, whereas the flow almost maintains its velocity on the sides of the nose. Two pairs of vortices are formed because of the flow velocity gradient. Without an interaction with the flow from the bogies, the strengths of the upper two pairs of vortices, which form due to the nose geometry, decrease rapidly in the wake, whereas the strength of the lower pair of vortices, which form as a result of the cowcatcher, is maintained for a longer distance in the wake.

Unlike in the simplified model, bogies exist in the real model and have two main effects on the external flow of the train. First, the adjacent flow is enhanced by the bogies, especially by the last bogie; this greatly enhances the wake flow of the real train model. Second, strong perturbances are also introduced into the flow of the real train model by the bogies, resulting in greater unsteadiness in the wake flow. 
Therefore, on the basis of the geometry of the simplified model, the existence of bogies in the real model, especially the last one, introduces strong perturbances into the wake flow, which results in the significant enhancement of the lower pair of vortices (in the wake flow field of the simplified model). Subsequently, these two vortices become larger and they affect a broader range, resulting in greater unsteadiness and periodicity of the wake flow of the real model. However, the effects of the bogies on the upper two pairs of vortices (in the wake flow field of the simplified model) are comparatively weaker. These four vortices would be inherently damped or merged with the strong lower vortices. Consequently, in the real model, the wake flow, which is enhanced by the perturbances of the bogies, is characterized by two large counter-rotating streamwise vortices, which are generated by the cowcatcher of the trailing car.

\section{Conclusions}

In this study, we investigated the wake flows of three CRH380A high-speed train models with different degrees of simplification, i.e., the real model with bogies on the bottom of the train, the semi-simplified model with only the last bogie, and the simplified model without any bogies; a numerical study using the IDDES method and the SST $k-\omega$ turbulence model was conducted. Analyses of the aerodynamic forces, train-induced slipstream, and TKE were conducted to determine the effects of the bogies on the external flow of the high-speed train. It was found that the existence of the bogies on the bottom of the high-speed train, especially the last bogie, not only enhanced the wake flow but also introduced large perturbances into the wake flow.

Moreover, the generation and evolution of the vortices in the wake flows of the three high-speed train models were obtained by analyzing the instantaneous flow fields and decomposing the wake flows using the DMD method. The results showed that three pairs of vortices occurred in the wake flow of the simplified high-speed train model. The lower pair of counter-rotating and alternately changing vortices was generated by the cowcatcher, whereas the upper pairs of alternately changing and rapidly damping vortices were generated by the trailing nose of the train. In the real high-speed train model, a pair of large, counter-rotating streamwise vortices occurred in the wake flow field. Comparisons of the characteristics of the trailing vortices of the real and simplified model were conducted to determine the detailed mechanism of generation and evolution of the trailing vortices in a real high-speed train. It was demonstrated that the pair of large, counter-rotating streamwise vortices was generated by the cowcatcher of the trailing car and was enhanced by the perturbances introduced by the bogies on the bottom of the train. Furthermore, it is also concluded that the geometry of bogies should not be neglected during the analysis of the wake flow of high-speed trains in experiments or numerical simulations because bogies are an important influencing factor on the generation and evolution of trailing vortices.

Author Contributions: Conceptualization: D.G. and G.Y.; analysis and writing: W.L.; validation: Z.Z. and D.C.; review: D.G. and G.Y.

Funding: The project was supported by the National Key R\&D Program of China (2016YFB1200506-22) and the Strategic Priority Research Program of the Chinese Academy of Sciences (XDB22020100).

Conflicts of Interest: The authors declare no conflict of interest.

\section{References}

1. Baker, C.J.; Dalley, S.J.; Johnson, T.; Quinn, A.; Wright, N.G. The slipstream and wake of a high-speed train. Proc. Inst. Mech. Eng. Part F: J. Rail Rapid Transit 2001, 215, 83-99. [CrossRef]

2. Baker, C.J. The flow around high-speed trains. J. Wind Eng. Ind. Aerod. 2010, 98, 277-298. [CrossRef]

3. Baker, C.J.; Quinn, A.; Sima, M.; Hoefener, L.; Licciardello, R. Full scale measurement and analysis of train slipstreams and wakes: Part1 ensemble averages. Proc. Inst. Mech. Eng. Part F: J. Rail Rapid Transit 2014, 228, 451-467. [CrossRef]

4. Rocchi, D.; Tomasini, G.; Schito, P.; Somaschini, C. Wind effects induced by high speed train pass-by in open air. J. Wind Eng. Ind. Aerod. 2018, 173, 279-288. [CrossRef] 
5. Bell, J.R.; Burton, D.; Thompson, M.C.; Herbst, A.H.; Sheridan, J. Wind tunnel analysis of the slipstream and wake of an HST. J. Wind Eng. Ind. Aerod. 2014, 134, 122-138. [CrossRef]

6. Bell, J.R.; Burton, D.; Thompson, M.C.; Herbst, A.H.; Sheridan, J. Flow topology and unsteady features in the wake of a generic high-speed train. J. Fluids Struct. 2016, 61, 168-183. [CrossRef]

7. Bell, J.R.; Burton, D.; Thompson, M.C.; Herbst, A.H.; Sheridan, J. The effect of tail geometry on the slipstream and unsteady wake structure of high-speed trains. Exp. Therm. Fluid Sci. 2017, 83, 215-230. [CrossRef]

8. Weise, M.; Schober, M.; Orellano, A. Slipstream velocities induced by trains. In Proceedings of the WSEAS International Conference on Fluid Mechanics and Aerodynamics, Elounda, Greece, 21-23 August 2006; pp. 26-28.

9. Yao, S.B.; Sun, Z.X.; Guo, D.L.; Chen, D.W.; Yang, G.W. Numerical study on wake characteristics of high-speed trains. Acta Mech. Sinica-Prc. 2013, 29, 811-822. [CrossRef]

10. Schulte-Werning, B.; Heine, C.; Matschke, G. Unsteady Wake Flow Characteristics of High-Speed Trains. Proc. Appl. Math. Mech. 2003, 2, 332-333. [CrossRef]

11. Xia, C.; Wang, H.F.; Shan, X.Z.; Yang, Z.G.; Li, Q.L. Effects of ground configurations on the slipstream and near wake of a high-speed train. J. Wind Eng. Ind. Aerod. 2017, 168, 177-189. [CrossRef]

12. Pii, L.; Vanoli, E.; Polidoro, F.; Gautier, S.; Tabbal, A. A full scale simulation of a high-speed train for slipstream prediction. In Proceedings of the Transport Research Arena, Paris, France, 14-17 April 2014.

13. Hemida, H.; Gil, N.; Baker, C.J. LES of the slipstream of a rotating train. J. Fluids Eng. 2010, 132, 051103. [CrossRef]

14. Hemida, H.; Baker, C.J.; Gao, G.J. The calculation of train slipstreams using large-eddy simulation. Proc. Inst. Mech. Eng. Part F: J. Rail Rapid Transit 2014, 228, 25-36. [CrossRef]

15. Muld, T.W.; Efraimsson, G.; Henningson, D.S. Flow structures around a high-speed train extracted using Proper Orthogonal Decomposition and Dynamic Mode Decomposition. Comput. Fluids 2012, 57, 87-97. [CrossRef]

16. Xia, C.; Wang, H.F.; Bao, D.; Yang, Z.G. Unsteady flow structures in the wake of a high-speed train. Exp. Therm. Fluid Sci. 2018, 98, 381-396. [CrossRef]

17. Muld, T.W.; Efraimsson, G.; Henningson, D.S. Wake characteristics of high-speed trains with different lengths. Proc. Inst. Mech. Eng. Part F: J. Rail Rapid Transit 2014, 228, 333-342. [CrossRef]

18. Zhang, J.; Wang, J.B.; Wang, Q.X.; Xiong, X.H.; Gao, G.J. A study of the influence of bogie cut outs' angles on the aerodynamic performance of a high-speed train. J. Wind Eng. Ind. Aerod. 2018, 175, 153-168. [CrossRef]

19. Wang, S.B.; Bell, J.R.; Burton, D.; Herbst, A.H.; Sheridan, J.; Thompson, M.C. The performance of different turbulence models (URANS, SAS and DES) for predicting high-speed train slipstream. J. Wind Eng. Ind. Aerod. 2017, 165, 46-57. [CrossRef]

20. Huang, S.; Hemida, H.; Yang, M. Numerical calculation of the slipstream generated by a CRH2 high-speed train. Proc. Inst. Mech. Eng. Part F: J. Rail Rapid Transit 2016, 230, 103-116. [CrossRef]

21. Schmid, P.J. Dynamic mode decomposition of numerical and experimental data. J. Fluids Mech. 2010, 656, 5-28. [CrossRef]

22. Jovanović, M.R.; Schmid, P.J.; Nichols, J.W. Sparsity-promoting dynamic mode decomposition. Phys. Fluids 2014, 26, 561-571. [CrossRef]

23. Schmid, P.J. Application of the dynamic mode decomposition to experimental data. Exp. Fluids 2011, 50, 1123-1130. [CrossRef]

24. Wind Tunnel Test Report for a New Generation of High-Speed Trains; CRRC Qingdao Sifang Co., Ltd.: Qingdao, China, 2009.

25. Schlichting, H. Boundary Layer Theory; Science Press: Beijing, China, 1988.

26. Zhang, Q.S.; Liu, Y.Z.; Wang, S.F. The identification of coherent structures using proper orthogonal decomposition and dynamic mode decomposition. J. Fluids Struct. 2014, 49, 53-72. [CrossRef]

27. Ahmed, S.R. Influence on base slant on the wake structure and drag of road vehicles. J. Fluid Eng.-T ASME 1983, 105, 429-434. [CrossRef]

(C) 2019 by the authors. Licensee MDPI, Basel, Switzerland. This article is an open access article distributed under the terms and conditions of the Creative Commons Attribution (CC BY) license (http:/ / creativecommons.org/licenses/by/4.0/). 